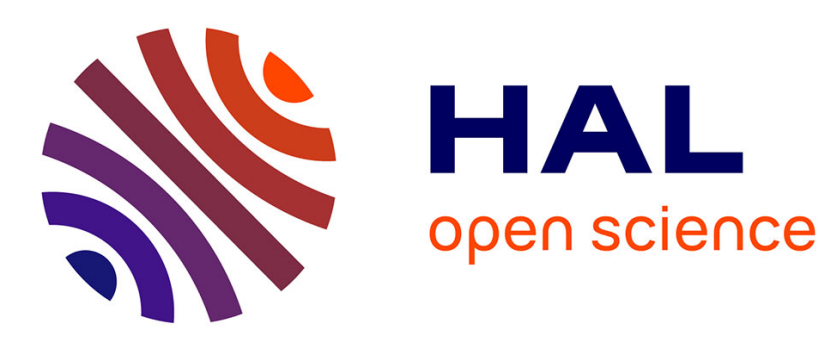

\title{
Control of confined vortex breakdown with partial rotating lids
}

\author{
Lewis Mununga, David Lo Jacono, Jens N. Sorensen, Thomas Leweke, Mark \\ C. Thompson, Kerry Hourigan
}

\section{- To cite this version:}

Lewis Mununga, David Lo Jacono, Jens N. Sorensen, Thomas Leweke, Mark C. Thompson, et al.. Control of confined vortex breakdown with partial rotating lids. Journal of Fluid Mechanics, 2014, vol. 738, pp. 5-33. 10.1017/jfm.2013.596 . hal-01092035

\section{HAL Id: hal-01092035 https://hal.science/hal-01092035}

Submitted on 8 Dec 2014

HAL is a multi-disciplinary open access archive for the deposit and dissemination of scientific research documents, whether they are published or not. The documents may come from teaching and research institutions in France or abroad, or from public or private research centers.
L'archive ouverte pluridisciplinaire HAL, est destinée au dépôt et à la diffusion de documents scientifiques de niveau recherche, publiés ou non, émanant des établissements d'enseignement et de recherche français ou étrangers, des laboratoires publics ou privés. 


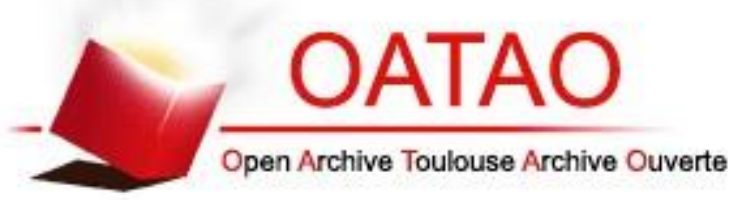

Open Archive Toulouse Archive Ouverte (OATAO)

OATAO is an open access repository that collects the work of Toulouse researchers and makes it freely available over the web where possible.

This is an author-deposited version published in: http://oatao.univ-toulouse.fr/ Eprints ID: 12211

Identification number: DOI : 10.1017/jfm.2013.596

Official URL: http://dx.doi.org/10.1017/jfm.2013.596

\section{To cite this version:}

Mununga, Lewis and Lo Jacono, David and Sorensen, Jens N. and Leweke, Thomas and Thompson, Mark C. and Hourigan, Kerry Control of confined vortex breakdown with partial rotating lids. (2014) Journal of Fluid Mechanics, vol. 738 . pp. 5-33. ISSN 0022-1120

Any correspondence concerning this service should be sent to the repository administrator: staff-oatao@inp-toulouse.fr 


\title{
Control of confined vortex breakdown with partial rotating lids
}

\author{
L. Mununga ${ }^{1}$, D. Lo Jacono ${ }^{1}$, J. N. Sørensen ${ }^{2}$, T. Leweke ${ }^{3}$, \\ M. C. Thompson ${ }^{1,4} \dagger$ and K. Hourigan ${ }^{1,4}$ \\ ${ }^{1}$ Fluids Laboratory for Aeronautical and Industrial Research (FLAIR), \\ Department of Mechanical Engineering, Monash University, Melbourne, Victoria 3800, Australia \\ ${ }^{2}$ Department of Mechanical Engineering, Technical University of Denmark, Lyngby, DK-2800, Denmark \\ ${ }^{3}$ Institut de Recherche sur les Phénomènes Hors Équilibre (IRPHÉ), UMR 6594 CNRS / Universités \\ Aix-Marseille I \& II, 49 rue Frédéric Joliot-Curie, B.P. 146, F-13384 Marseille CEDEX 13, France \\ ${ }^{4}$ Division of Biological Engineering, Monash University, Melbourne, Victoria 3800, Australia
}

Experiments were conducted to determine the effectiveness of controlling vortex breakdown in a confined cylindrical vessel using a small rotating disk, which was flush-mounted into the opposite endwall to the rotating endwall driving the primary recirculating flow. The results show that the control disk, with relatively little power input, can modify the azimuthal and axial flow significantly, changing the entire flow structure in the cylinder. Co-rotation was found to precipitate vortex breakdown onset whereas counter-rotation delays it. Furthermore, for the Reynolds-number range over which breakdown normally exists, co-rotation increases the bubble radial and axial dimensions, while shifting the bubble in the upstream direction. By contrast, counter-rotation tends to reduce the size of the bubble, or completely suppress it, while shifting the bubble in the downstream direction. These effects are amplified substantially by the use of larger control disks and higher rotation ratios. A series of numerical simulations close to the onset Reynolds number reveals that the control disk acts to generate a rotation-rate-invariant local positive or negative azimuthal vorticity source away from the immediate vicinity of the control disk but upstream of breakdown. Advection of this source along streamlines modifies the strength of the azimuthal vorticity ring, which effectively controls whether the flow reverses on the axis, and thus, in turn, whether vortex breakdown occurs. The vorticity source generated by the control disk scales approximately linearly with rotation ratio and cubically with disk diameter; this allows the observed variation of the critical Reynolds number to be approximately predicted.

Key words: flow control, vortex breakdown, vortex flows

\section{Introduction}

The first observation of vortex breakdown in a cylindrical enclosure with one rotating endwall is credited to Vogel (1968). His experimental visualizations showed a recirculation region that appeared to resemble a bubble near the axis of rotation.

$†$ Email address for correspondence: mark.thompson@monash.edu 
Among other important early works on vortex breakdown in confined flows is the detailed investigation by Escudier (1984), who extended the works of Vogel (1968) and Ronnenberg (1977) by mapping regions of one, two and three vortex breakdowns, and regions that are steady and unsteady, as a function of Reynolds number based on the rotation velocity at the outside radius of the endwall and cylinder aspect ratio.

Vortex breakdown is important in a variety of technical areas such as aerodynamics (open-flow applications) and combustion (semi-enclosed applications). Interestingly, in real-life applications, the presence of vortex breakdown may be detrimental or beneficial. It is therefore of considerable importance to investigate methods of controlling vortex breakdown so that it can be enhanced where advantageous and suppressed where detrimental. Cell or tissue growth research in bioreactors is a very good example of a real-life application where vortex breakdown control may be important. Current research is trying to locate scaffolds for cell proliferation in a region of a cylindrical spinning-disk bioreactor where the flow is laminar, to exploit the low shear stress and significant residence times for nutrient injection (Yu et al. 2005b, 2009; Dusting, Sheridan \& Hourigan 2006). Additionally, variations of rotatingdisk bioreactors are being tested for their ability to provide a suitable environment for the growth and adhesion of cells (Thouas, Sheridan \& Hourigan 2007; Liow et al. 2009). It is speculated that laminar flow inside a spinning-disk bioreactor, and, in particular, the vortex breakdown bubble, may constitute an ideal environment for the proliferation of certain cell types. The method of controlling vortex breakdown being proposed here may represent an attractive way of manipulating the flow environment without excessive additional energy input (Mununga 2005).

Vortex breakdown control in closed cylinders has not been investigated as widely as breakdown control of external flows. In fact, most of the literature on confined flow vortex breakdown control has only appeared in the last two decades. The main techniques employed to control vortex breakdown have made use of temperature gradient (Herrada \& Shtern 2003a), co-rotation (Valentine \& Jahnke 1994; Bhattacharyya \& Pal 1998) and counter-rotation (Roesner 1990) of the end walls, the addition of near-axis swirl modification (Husain, Shtern \& Hussain 2003; Lo Jacono et al. 2008), upstream modulation (Lopez et al. 2008), tilted endwalls (Meunier \& Hourigan 2013), conical endwalls (Yu et al. 2006) and even small density effects due to the introduction of dye (Ismadi et al. 2011).

Roesner (1990) experimentally investigated vortex breakdown in the confined region of a cylinder with independently rotating top and bottom endwalls. He first investigated the case where the top lid was rotating while the bottom was at rest, and later considered the co- and counter-rotation cases where both endwalls were spun. Roesner noted that, if the Reynolds number of the top endwall was slightly below the critical value where the first breakdown bubble is normally expected to occur, a slight co-rotation of the bottom endwall immediately created a recirculation motion along the axis of rotation. On the other hand, if the top-disk Reynolds number was slightly above this critical value, a slow counter-rotation of the bottom endwall caused the recirculation region to disappear.

Co-rotation of the endwalls has also been employed by Bhattacharyya \& Pal (1998) to control vortex breakdown in a laminar swirling flow inside a cylindrical container. They numerically modelled the flow by inducing a slight co-rotation $(0<\varepsilon<0.5)$ of the top and bottom endwalls. Here, $\varepsilon$ is the angular velocity ratio, defined as the ratio between the angular velocities of the top $\left(\Omega_{t}\right)$ and the bottom $\left(\Omega_{b}\right)$ endwalls. They found that slight co-rotation of the upper endwall promotes the onset of vortex breakdown. This means that, for the upper and lower endwalls rotating in the same 
direction, the critical Reynolds number for vortex breakdown onset was considerably reduced. Another finding from this study was that the vortex breakdown bubble appeared near the faster-moving endwall, which in this case was the bottom endwall. That finding is rather unexpected considering that, in cases where only one endwall rotates, the vortex breakdown almost always appears closer to the stationary endwall (Escudier 1984).

Valentine \& Jahnke (1994) predicted numerically the flow field inside a cylindrical container induced by the rotation of two endwalls at the same rate (i.e. $\varepsilon=1.0$ ). They found different types of recirculation bubbles depending on the combination between the Reynolds number and the aspect ratio $(H / R)$. They reported that, for high-aspect-ratio cylinders and relatively low Reynolds numbers, slender recirculation bubbles appeared on the axis of the container. At high Reynolds numbers, they noted the formation of a toroidal vortex around an axial vortex core. The important point to note here, although not emphasized by Valentine \& Jahnke, is that co-rotation of the two endwalls was conducive to vortex breakdown formation. With co-rotation, vortex breakdown onset occurred at lower Reynolds numbers than it would have for the cases with rotation of only one endwall.

Subsequently, Okulov, Sørensen \& Voigt (2004) studied the effect of co- and counter-rotation of both top and bottom endwalls in a closed cylindrical container. They were able to generate a vortex breakdown while rotating the two endwalls at the same rate $(\varepsilon=1.0)$. Although this study was mainly concerned with the onset of vortex breakdown rather than the control aspect, it nevertheless demonstrated that co-rotation of the two endwalls could still produce vortex breakdown. At this stage, it is worth mentioning that the vortex breakdown control methods reported so far have considered mainly the co-rotation case with relatively small rotation ratios, except in the case of Valentine \& Jahnke (1994) and Okulov et al. (2004), who considered the case of two endwalls rotating at the same rate. Other studies have also been devoted to the onset of vortex breakdown (Roesner 1990). More expansive investigations are therefore suggested to extend the range of rotation ratios and also to examine the control of vortex breakdown using both co-rotation and counter-rotation of the opposing endwall.

Bhattacharyya \& Pal (1999) implemented vortex breakdown control by the spin-up and spin-down processes. The base flow was generated by rotating the lower endwall or by co-rotating both endwalls. The spin-up process was achieved by impulsively rotating the upper endwall with smaller angular velocity in the same direction as the lower endwall. The spin-down process was achieved by abruptly bringing to rest the top endwall, which was co-rotating with small rotation ratio $(\varepsilon \leqslant 0.2)$. Before the spin-down process, a separation bubble was present, whereas before the spin-up process, the flow was free of a separation bubble. Bhattacharyya \& Pal (1999) also reported that during the transient period, while the bubble was shrinking, it was moving towards the slow-moving upper endwall. They concluded that the spin-up and spin-down processes can effectively generate and dissipate a separation bubble in a closed cylinder filled with liquid.

The concept of using a partially rotating disk within the endwall has been considered by a few researchers (Piva \& Meiburg 2005; Yu et al. 2005a,b, 2007). It was found that the cylinder-to-disk ratio has a dominant effect on the appearance of vortex breakdown, and that varying the size of the rotating disk could control vortex breakdown behaviour.

Husain et al. (2003) employed the concept of near-axis swirl addition using a small centrally located rod rotating independently of the bottom or rotating endwall. To 
control vortex breakdown, they rotated the rod either in the same direction as the rotating endwall or in the opposite direction, the top endwall being kept stationary. The central rod size was chosen to be close to the vortex core diameter. They showed that the addition of swirl near the axis of rotation was an effective way of controlling vortex breakdown. Essentially, their findings were that co-rotating the small rod with respect to the rotating endwall retained a steady flow while suppressing vortex breakdown bubbles, whereas counter-rotating induced centrifugal instability, resulting in vortex breakdown enhancement. They reasoned that co-rotation of the small rod decreased the unfavourable pressure gradient around the axis and thereby suppressed the vortex breakdown bubble. For the case where the small rod was counter-rotated, Husain et al. (2003) explained that the additional swirl caused the generation of a centrifugal instability, leading to unsteady flow conditions favourable to formation or enhancement of vortex breakdown bubbles. Herrada \& Shtern (2003b) also considered the effects of near-axis swirl and temperature gradients on the breakdown bubble. Clearly, temperature gradients are relevant to bioreactor applications. Later, Lo Jacono et al. (2008) examined the effect of rotating rods of different lengths, both experimentally and numerically. The rod length was found to have a significant effect on breakdown, and this was analysed and interpreted in terms of additional vorticity source terms caused by the introduction of the small rod. Cabeza et al. (2010) examined the effect of the diameter of a non-rotating rod, which was also found to exert a strong influence on breakdown. These methods, although effective, are at least partially intrusive, with the rod passing through the centre of the vortex core and vortex breakdown, although a small-diameter stationary rod has only a minor effect on the vortex breakdown patterns. Ideally, it would be preferable to use a method that clearly does not interfere with the flow structure being examined. This consideration is addressed by the current study.

Recently Shtern, del Mar Torregrosa \& Herrada (2012) examined vortex breakdown from the effect of downstream swirl decay. As is seen later, this framework allows the observed opposite effects of a rotating rod and a small embedded disk on vortex breakdown enhancement/decay to be predicted. Co-rotation of the disk enhances breakdown, while co-rotation of the rod delays it.

The onset of vortex breakdown is usually expressed in terms of a critical swirl number, or the ratio between the swirl velocity and the axial velocity locally in a swirling jet (e.g. Squire 1960; Benjamin 1962; Keller, Egli \& Exley 1985; Spall, Gatski \& Gresch 1987; Billant, Chomaz \& Huerre 1998). This suggests the approach to controlling vortex breakdown through modifying the swirl number by the addition of swirl (co-rotating or counter-rotating) using a small independently controlled rotating disk (figure 1). In addition, the rotation of the small disk may also affect the axial flow along the swirl axis. In this paper, the effects of co-rotation and counterrotation of a small disk on vortex breakdown are examined and quantified, leading to a better understanding of the capabilities of this approach.

The overall aim of this study is to quantify the effectiveness of a proposed nonintrusive method of controlling vortex breakdown using a set of small embedded disks, and to investigate the mechanisms leading to its efficacy. The research described here documents large changes to the interior flow structure induced by localized flow modification at the upstream boundary. Furthermore, numerical solutions allow the additional positive or negative azimuthal vorticity source generated by the control disk to be examined in detail; this source in turn is responsible for the modification to the downstream flow delaying or enhancing breakdown. 


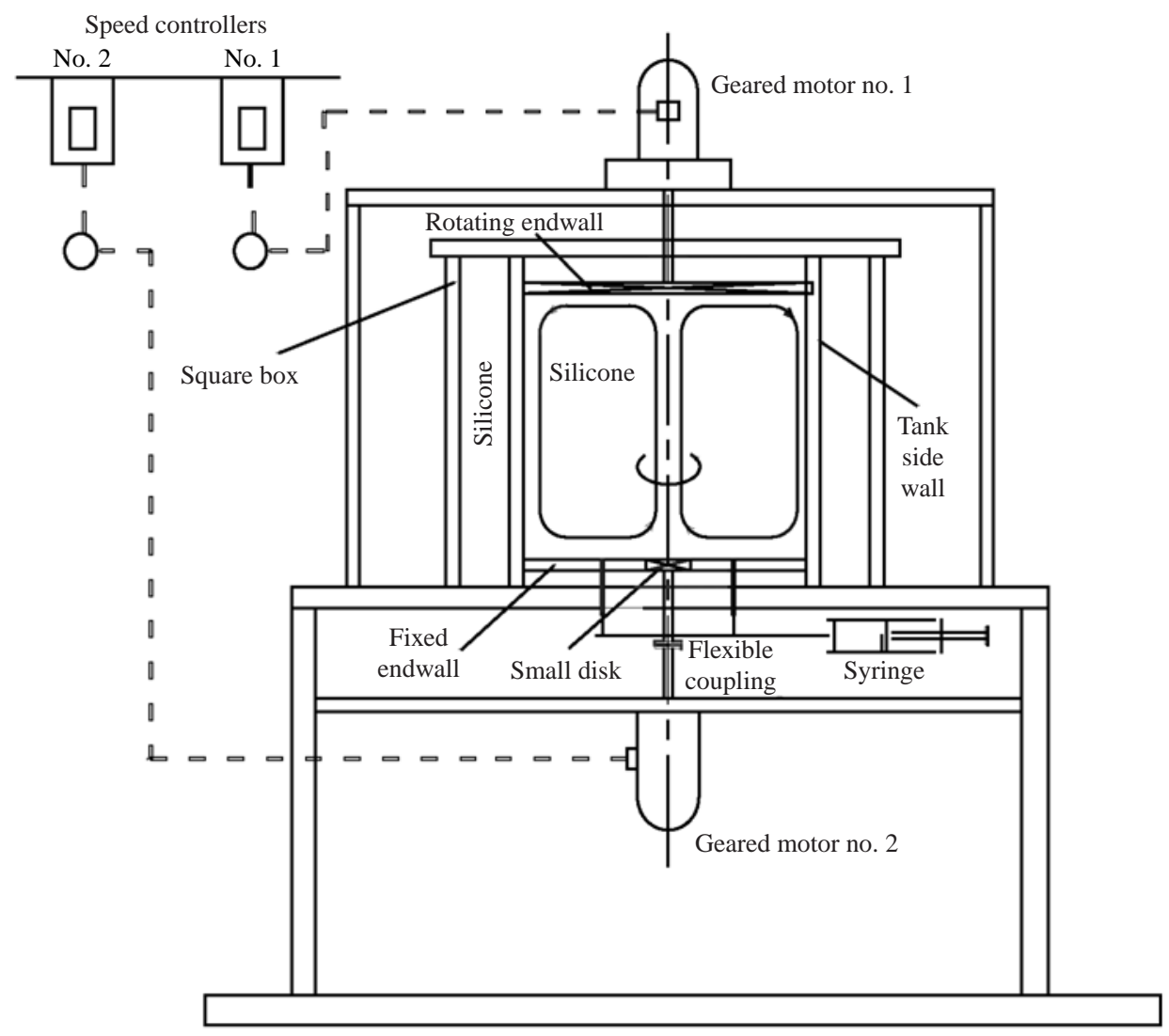

FIGURE 1. Experimental rig.

\section{Experimental methods}

\subsection{Experimental set-up and data processing}

A schematic of the experimental rig is shown in figure 1. The rig consisted of a cylindrical Perspex tube with an inner radius $R=243 \mathrm{~mm}$ filled with silicone oil (Shin-Etsu), which had an absolute viscosity $\mu=0.5 \mathrm{~kg} \mathrm{~m}^{-2} \mathrm{~s}$ and a density $\rho=969 \mathrm{~kg} \mathrm{~m}^{-3}$ at $25^{\circ} \mathrm{C}$. The aspect ratio $H / R=2$ was fixed, where $H$ is the height of the fluid column between the two endwalls and $R$ is also equal to the radius of the rotating endwall (or top endwall). In the bottom endwall, a recess was precisionmachined to accommodate small disks (or control disks) of different sizes. Table 1 shows the dimensions of the five different small disks employed in this study. Each small disk was flush-mounted axisymmetrically into the non-rotating endwall.

The flow structure within the working section was visualized by shining a thin sheet of light $(\approx 5 \mathrm{~mm})$ emanating from twin $500 \mathrm{~W}$ floodlights mounted on a stand holder raised approximately to the same level as the centre of the working section. To highlight the flow structures, a small quantity of silicone oil mixed with seeding particles was slowly injected by means of a hypodermic syringe through two small holes in the fixed endwall. Photographs were taken using a high-resolution digital camera, and processed using Matlab image processing routines to determine the size and location of the bubble to an accuracy of better than $\pm 3 \%$. The rotating endwall 


$\begin{array}{lccc}\text { Disk } & \begin{array}{c}\text { Radius } r_{d} \\ (\mathrm{~mm})\end{array} & r_{d} / R & \begin{array}{c}\text { Effective area } \\ (\% \text { of large } \\ \text { disk })\end{array} \\ d_{1} & 12.5 & 0.0514 & 0.26 \\ d_{2} & 24.5 & 0.1008 & 1.02 \\ d_{3} & 37.0 & 0.1523 & 2.32 \\ d_{4} & 49.0 & 0.2016 & 4.07 \\ d_{5} & 74.5 & 0.3066 & 9.40\end{array}$

TABLE 1. Control disks used in the experiments.

and the control disk were independently driven by two electric motors via gearboxes. The motors were operated through frequency inverters.

From the viewpoint of vortex breakdown control, the above rig design offers a number of advantages over previous designs. First, it is possible to study the flow structure due to the combined rotation of the top endwall and control disk without introducing a foreign object into the working section, as was the case in the studies of Husain et al. (2003) and Lo Jacono et al. (2008). Second, the present control method looks more feasible and attractive than the use of two rotating endwalls (Valentine \& Jahnke 1994) because a small disk leads to a significantly reduced power requirement (Mununga 2005; Tan et al. 2009) for the second drive system and therefore a less bulky structure. (Note that the present and latter papers report on different aspects of this research programme. The numerical study by Tan et al. (2009) focuses on the power requirement of using a small disk for controlling breakdown. That paper only includes the single experimental result of the shift in vortex breakdown onset for disk $d_{3}$.) In addition, the small disk more directly targets the flow immediately upstream of the bubble, so it might be expected to be a more efficient means of flow control.

The seeding particles employed were S-HGS silver-coated hollow glass spheres. These are smooth borosilicate glass particles with a thin silver coating designed to increase the reflectivity and provide light scattering conducive to successful (even spectacular) experimental images. These particles had a mean particle size of approximately $10 \mu \mathrm{m}$ and a density of $1.4 \mathrm{~kg} \mathrm{~m}^{-3}$.

The rotational speeds of the rotating endwall and the small disk were determined from the corresponding frequency inverter readings. In addition, readings from a digital photo tachometer, of accuracy $\pm 0.05 \%$, were used to confirm the accuracy of the inverter. Based on the above figures, the uncertainties in $R e$ and $R e_{d}$ (the Reynolds numbers associated with the endwall and control-disk rotations) were calculated to be $\pm 3.5 \%$ and $\pm 2.5 \%$, respectively. As is usual for the spinning lid rig, the Reynolds number is given by $R e=\Omega_{D} R^{2} / \nu$, with $v$ the kinematic viscosity, and the smalldisk Reynolds number is defined by $R e_{d}=\Omega_{d} r^{2} / \nu$, where $r$ is the small-disk radius. The control to endwall disk angular velocity ratio, $\varepsilon=\Omega_{d} / \Omega_{D}$, had an uncertainty estimated to be $\pm 1 \%$.

As described above, the flow visualization photographs taken during the experiments were analysed in the Matlab environment to determine the radius, positions (heights) of upstream and downstream stagnation points and the centre of the primary vortex breakdown bubble. The centre of the main vortex breakdown was considered to be at the intersection between the horizontal line cutting the bubble at its largest radius and the centreline representing the swirl axis. While it was relatively easy to accurately determine the position of the upstream stagnation point, it was often more difficult to 
locate the downstream stagnation point, especially when the main breakdown bubble appeared open at the downstream end. This explains why relatively only few data related to the height of the downstream stagnation point are presented in some of the figures. In the results presented below, all lengths are non-dimensionalized by the cylinder height $(H=2 R)$. The normalized upstream and downstream axial locations of the breakdown bubble are denoted by $z_{u}$ and $z_{d}$, respectively, and the normalized radial size by $r_{b}$.

\subsection{Calibration}

The first task was to determine accurately the viscosity of the working fluid and its variation with temperature. This was prompted by the fact that the viscosity specified by the manufacturer was only provided for a temperature of $25^{\circ} \mathrm{C}$, and was also of low quoted accuracy (nominal value of $500 \mathrm{~mm}^{2} \mathrm{~s}^{-1} \pm 5 \%$ ). To determine accurately the correct fluid temperature, two type $\mathrm{T}$ thermocouples were attached to the cylindrical tank to enable direct measurement of the temperature inside the working section. The temperature was sampled at two locations: one between the rotating endwall and the horizontal mid-plane, and the other in the bottom half of the vessel. The other ends of the thermocouples were coupled to a switch, which in turn was connected to a calibrated Anritsu handheld digital thermometer with an accuracy $\pm 0.5 \%$. In addition, the accuracy of the present results depended in part on how accurately the fluid viscosity was determined at each run. Calibration was performed using a laboratory rheometer fitted with a cup where the fluid was placed and a disk attached to a spindle. It was determined that the average absolute viscosity of the experimental fluid varied with temperature according to $\mu=-0.0107 T+0.7672 \mathrm{~kg} \mathrm{~m}^{-2} \mathrm{~s}$ with an accuracy of $\pm 0.5 \%$. This expression was used to determine the fluid viscosity during the experiments.

\subsection{Description of experiments}

\subsubsection{Vortex breakdown without control}

Initial experiments were performed to validate the experimental rig using published results. Subsequently, those results defined the baseline data set for comparing results generated from the vortex breakdown control experiments. The endwall rotational speed was steadily incremented from the vortex breakdown onset level $(R e=1440)$ to approximately $R e=3000$. At each step, after equilibrium was re-established, digital photographs were taken and transferred to the laboratory computer for processing in Matlab. For these experiments, the small disk was kept stationary.

\subsubsection{Vortex breakdown onset}

The purpose of this set of experiments was to quantify the effect of co- and counter-rotation of the small disks on the onset of vortex breakdown in terms of the Reynolds number of the rotating endwall. (For the remainder of the paper, coand counter-rotation of the control disk refers to rotation in the same and opposite direction to the endwall rotation, respectively.) From a practical point of view, vortex breakdown onset was defined as the flow state when the smallest visible bubble was observed along the swirl axis. With the rotating endwall spinning just at the speed at which vortex breakdown occurs, corresponding to $R e \approx 1440$, the small disk was made to rotate with the minimum possible angular speed, which in the present case was $\pm 5.95 \mathrm{rad} \mathrm{s}^{-1}$. The flow structure was then observed: (i) for the co-rotation case, the vortex breakdown bubble was observed to grow, hence the endwall rotation had to be carefully and gradually decreased until the onset state was re-established; (ii) whereas 
for the case of counter-rotation, the endwall rotation rate had to be gradually increased to once again achieve the critical state of vortex breakdown onset. The small-disk rotation rate was subsequently incremented and the endwall angular velocity adjusted accordingly so as to reach the state of vortex breakdown. In all cases, the experiment would continue until it was no longer possible to sustain the vortex breakdown state; this depended on the size of the control disk.

\subsubsection{Vortex breakdown control}

Another set of experiments was designed to study the effect of co-rotation and counter-rotation of the control disk on established vortex breakdown bubbles. In these experiments, the endwall was spun at a constant angular speed while the small disk was rotated in one direction, and photographs of the modified flow structure were taken to determine the modified bubble dimensional parameters. The speed of the control disk was increased in small steps while maintaining the same speed of the endwall. In practice, it was necessary to make small adjustments to the endwall rotation rate to account for the fractional variations in fluid temperature in order to maintain a constant $R e$. The rotational direction of the small disk was then reversed, while still keeping the endwall rotation constant, and the experiment proceeded as described above.

\section{Results}

The main experimental results presented in the following sections consist of photographs showing visualizations of different flow patterns inside the closed cylinder together with the observed relationships between various vortex breakdown variables derived from the images. The dependent variables include the normalized radial size of the breakdown bubble, and the axial location of the stagnation points and the centre of the breakdown bubble. The key independent variables are the rotating-endwall Reynolds number and the rotation ratio. Most of the results include co-rotation and counter-rotation data in order to ascertain and compare their separate effects on the variables of interest. Results for the experiments with no control are presented first, followed by those concerned with the control of vortex breakdown.

\subsection{One endwall rotation}

The first set of results from the experiments was for the case with the endwall rotating at a constant angular velocity and the control disk was stationary. As indicated above, these were primarily used to validate the current experiments, but also to provide baseline results for the control experiments.

\subsubsection{Flow visualization}

Figure 2 illustrates the flow visualization results for the experiments in which only the endwall was rotated at a constant angular velocity $\left(\Omega_{D}\right)$ - this case will be referred to as the 'no-control' case. Note that, in line with many previous experiments, the visualizations presented in this paper of the nominally axisymmetric bubbles are not fully axisymmetric and sometimes show open bubbles. This can be a result of visualization material injection offset (e.g. Hourigan, Graham \& Thompson 1995; Brøns, Thompson \& Hourigan 2009) or very slight misalignment of vessel components (Ventikos 2002; Thompson \& Hourigan 2003; Brøns, Shen \& Sørensen 2007). The well-known experimental results of Escudier (1984), for an aspect ratio of $H / R=2.0$, were used for validation. The following observations can be made: the onset of vortex breakdown occurred at $R e=1440$ (figure $2 a$ ); at $R e=1476$, there appeared a small 
(a)

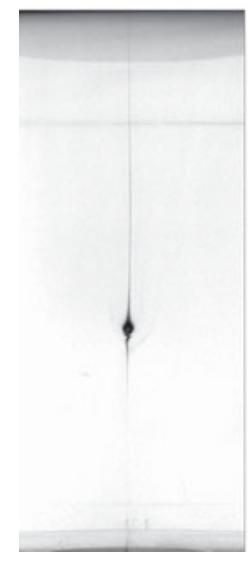

(b)

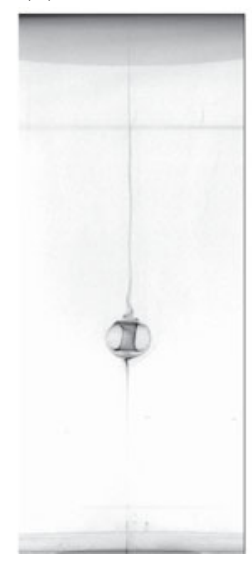

(c)

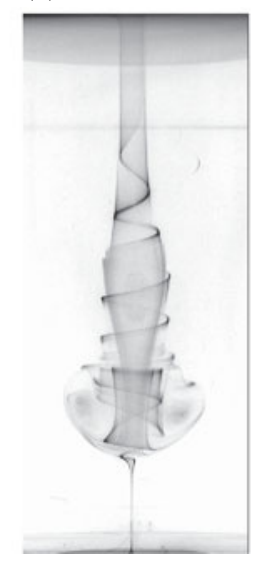

(d)

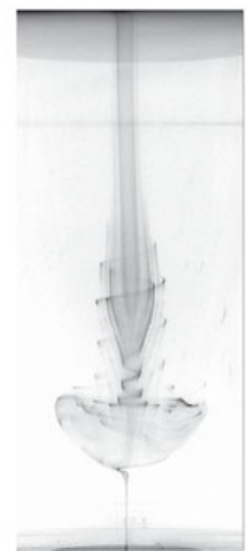

(e)

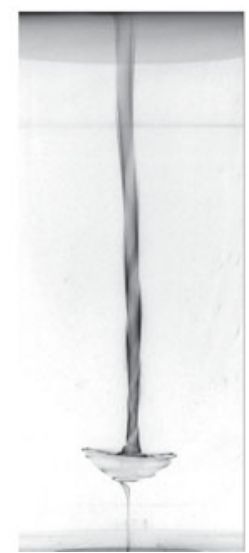

FIGURE 2. Flow visualization of vortex breakdown with no control at various Reynolds numbers: (a) $R e=1440$; (b) $R e=1476$; (c) $R e=2132$; (d) $R e=2474$; (e) $R e=2928$.

recirculation bubble (figure $2 b$ ); at $R e=2132$, the second breakdown bubble appeared downstream of the first bubble (figure 2c); as the Reynolds number reaches 2474, the second recirculation bubble opened up at the downstream end and therefore ceased to be a (closed) breakdown bubble (figure 2d); and finally, at $R e=2928$, the second bubble completely disappeared while the first breakdown bubble became deformed, taking the shape of a bowl supporting a downstream vortex core (figure $2 e$ ). Other results for $R e>3000$, not shown here, confirmed the total disappearance of the breakdown bubble. The above observations are in very good general agreement with those of Escudier (1984). More quantitative comparisons are provided below.

\subsubsection{Bubble radius}

The growth of the vortex breakdown bubble with respect to the rotating-endwall Reynolds number was examined and the results are presented in figure 3 . The bubble size was seen to increase, with corresponding increases in the Reynolds number until it reached a maximum value well beyond the two-bubble region. This was then followed by a rapid size reduction phase. From figure 3 , it can be seen that the maximum bubble size occurs in the range between $R e=2350$, just after the disappearance of the second bubble, and $R e=2700$, which is well into the second single bubble regime.

\subsubsection{Axial location of the bubble}

Figure 4 shows the variation of the axial location of the upstream, downstream and centre of the breakdown bubble with the rotating-endwall Reynolds number. The data of Fujimura et al. (2004) of the upstream stagnation point position are provided for comparison with the current results, primarily for quantitative validation purposes. The breakdown bubble was observed to migrate upstream, away from the rotating endwall, as the Reynolds number was increased. It is also important to note that, as the Reynolds number continued to increase, the rate of decrease of the upstream stagnation point height reduced, causing the upstream stagnation point position to asymptote, as depicted in figure 4 through the flattening of the bottom curve. The current results for the upstream stagnation point position were found to be within $2 \%$ of those of Fujimura et al. (2004) for the same aspect ratio $H / R=2.0$. The centre of the bubble was seen to have a characteristic movement similar to the upstream 


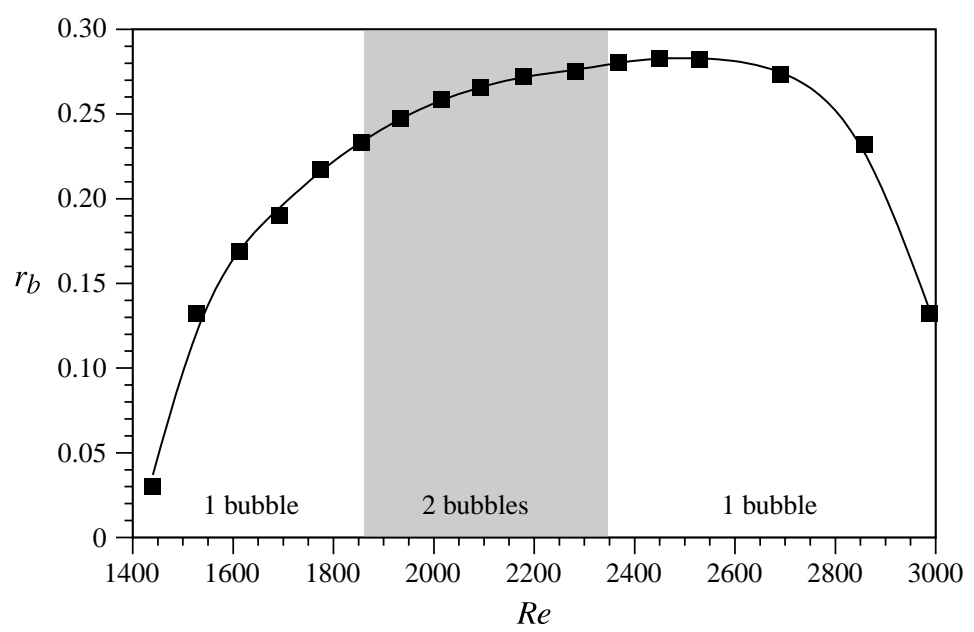

FIGURE 3. Evolution of the normalized radial size of the vortex breakdown bubble with respect to the rotating-endwall Reynolds number.

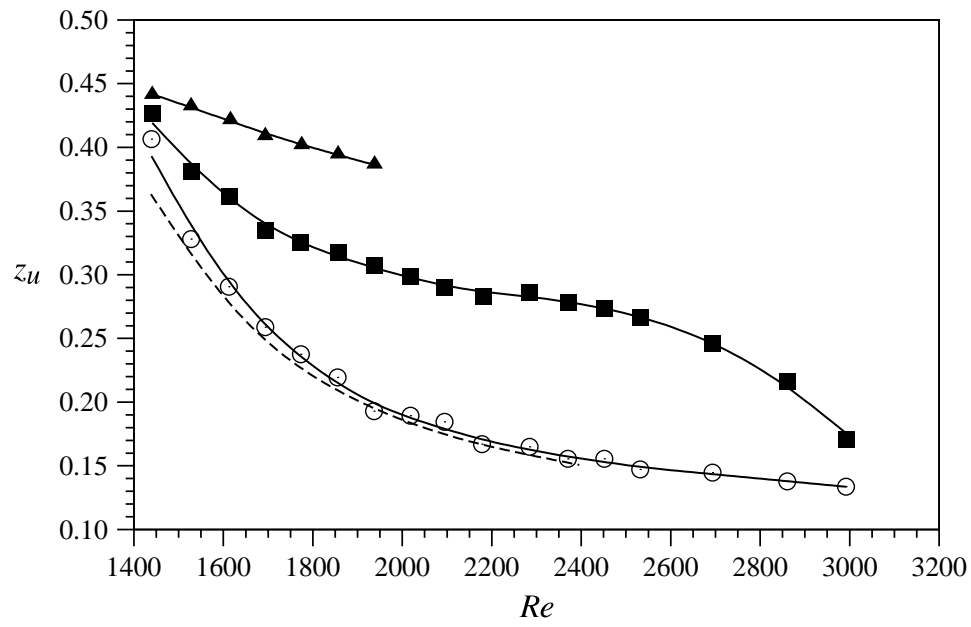

FIGURE 4. The solid lines indicate the axial location of the main vortex breakdown bubble for different endwall Reynolds numbers from the experiments. The lower, middle and upper curves show the positions of the upstream, centre and downstream extent of the breakdown bubble. The experimental data of Fujimura, Koyama \& Hyun (2004) for the upstream position, shown by the dashed line, are provided for comparison with the current experimental results.

stagnation point, except near the breakdown onset Reynolds number and the Reynoldsnumber range over which the bubble size begins to shrink, leading to its eventual disappearance. Furthermore, the data representing the migration of the downstream stagnation point also show a downward trend, indicating that the entire breakdown bubble was in fact moving towards the non-rotating endwall at the bottom of the vessel. As noted above, the fact that only few data points for the lower stagnation point position are shown was due to the difficulty in identifying these points as the 


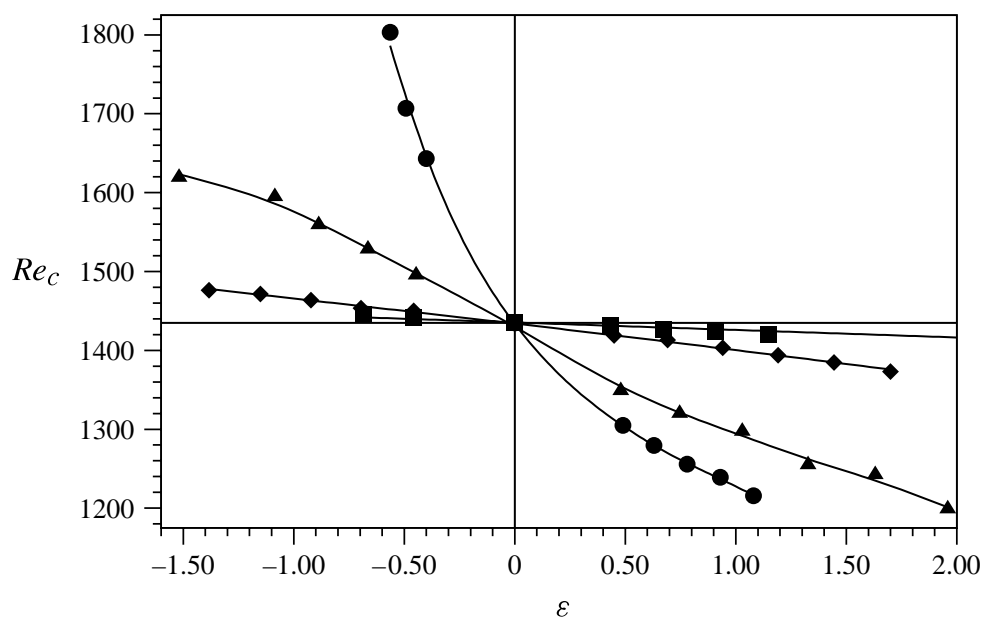

FIGURE 5. Variation of the critical Reynolds number $R e_{c}$ with rotation ratio $\varepsilon$. (Positive values indicate co-rotation and negative values indicate counter-rotation.) The effect of the small-disk size is shown for four different control-disk sizes: $d_{1}$, squares; $d_{2}$, diamonds; $d_{3}$, triangles; $d_{4}$, circles).

Reynolds number was increased; this difficulty was partly attributed to the fact that, beyond $R e>1850$, the second breakdown bubble appeared to emerge from inside the first bubble. The size of the bubble in the axial direction can also be deduced from the heights of the two stagnation points and the centre of the bubble at each Reynolds number. Therefore, as in the case of radial size, it can be seen that the bubble size in the axial direction tends to increase with rotational velocity of the top endwall, from the vortex breakdown onset to the time it reaches its maximum value $(2350<\operatorname{Re}<2700)$.

\subsection{Control of vortex breakdown with a small rotating disk}

As indicated, the proposed vortex breakdown control method consisted of co-rotating and counter-rotating a small control disk embedded in the otherwise stationary bottom endwall (figure 1). Experimental results for vortex breakdown control are presented below, beginning with results for vortex breakdown onset. Also, the effects of co- and counter-rotation, control-disk size and rotation ratio on the main breakdown bubble are investigated and quantified.

\subsubsection{Control of vortex breakdown onset}

Experiments were conducted to determine the shift in the critical Reynolds number $\left(R e_{c}\right)$ for vortex breakdown onset under the combined influence of the rotation of the top endwall and the control disk for all five disk diameters $\left(d_{1}, d_{2}, d_{3}, d_{4}\right.$ and $\left.d_{5}\right)$. However, experiments conducted with the control disk $d_{5}$ were unsatisfactory due to practical limitations: even at the smallest rotation rate obtainable using the bottom drive unit 2 (figure 1), the rotational effect of $d_{5}$ was too strong to generate or sustain the vortex breakdown onset state. This was true for both co-rotation and counterrotation. As a result, the planned experiments using this disk were discontinued.

Figure 5 displays the dependence of the critical Reynolds number on the rotation ratio and also the sense of rotation (co- or counter-rotation). In general, the results showed that, for co-rotation, the higher the rotation ratio, the lower the critical 
(a)

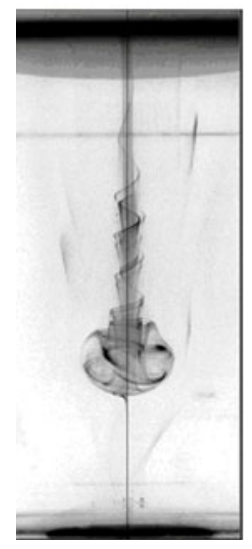

(b)

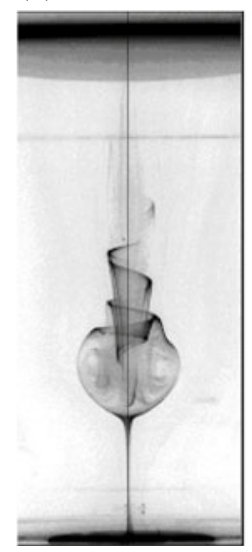

(c)

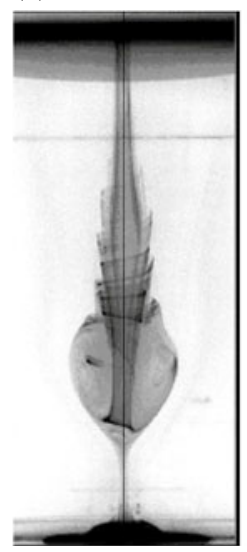

$(d)$

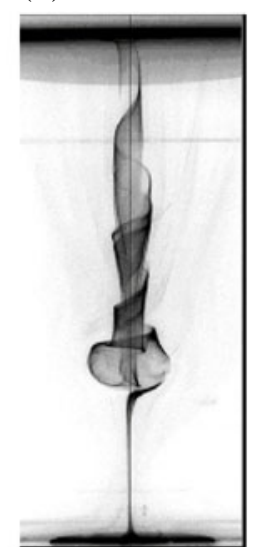

$(e)$

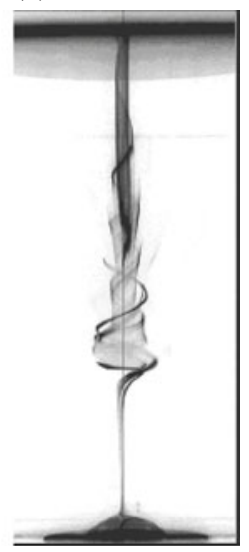

FIgURE 6. Flow visualization of vortex breakdown due to the top endwall rotation at $R e=1660$ and the effect of the small-disk $d_{3}$ rotation ratio $\varepsilon:(a)$ no control; $(b) \varepsilon=0.39$; (c) $\varepsilon=1.23 ;(d) \varepsilon=-0.39 ;(e) \varepsilon=-1.23$.

Reynolds number associated with the onset of vortex breakdown. However, the opposite was true when the control disk was counter-rotated relative to the rotating endwall. In addition, the results revealed that the smallest control disk $d_{1}$ only marginally altered the Reynolds number at which vortex breakdown first occurred; this is represented by an almost horizontal line in the graph. However, the biggest control disk, $d_{4}$ in this case, had a significant effect on the value of the critical Reynolds number, as shown by the steeply falling (co-rotation) and rising (counter-rotation) curves in figure 5. Some results using only the small disk $d_{3}$ have been reported previously by Mununga et al. (2004).

\subsubsection{Effects of co- and counter-rotation}

\section{(a) Flow visualization}

The results presented in figure 6 were obtained by rotating the top endwall at a constant angular velocity corresponding to $R e=1660$, while control was achieved by co-rotating and counter-rotating the control disk $d_{3}$. Figure 6 shows flow visualization pictures obtained when the rotating endwall and the control disk $d_{3}$ were co-rotated at $\varepsilon=0.39$ (figure $6 b$ ) and $\varepsilon=1.23$ (figure $6 c$ ), and counter-rotated at the same rates (figure $6 d, e$ ). Figure 6(a) represents the initial vortex breakdown bubble generated by rotating only the top endwall (i.e. for no control). The purpose of these experiments was to show how co-rotating and counter-rotating the control disk transformed the initial vortex breakdown. It was observed, as depicted in the images, that co-rotation of the control disk substantially increased the size of the breakdown bubble, whereas counter-rotation substantially decreased its size. Furthermore, the bubbles resulting from co-rotation appear stretched in the axial direction, with figure $6(c)$ showing a tendency for a second bubble to appear downstream of the first. By contrast, the bubbles generated under counter-rotation appear to shrink in the axial direction. Other results, not shown here, revealed that, for higher rotation ratios, counter-rotating $d_{3}$ leads to the complete disappearance of the breakdown bubble. It was also noted that, as the speed of the control disk increased, irrespective of the direction, another bubble started to form immediately on top of the smaller disk. This other bubble, referred to as the 'disk bubble', can be seen in figure 6(c) for co-rotation and figure 6(e) 


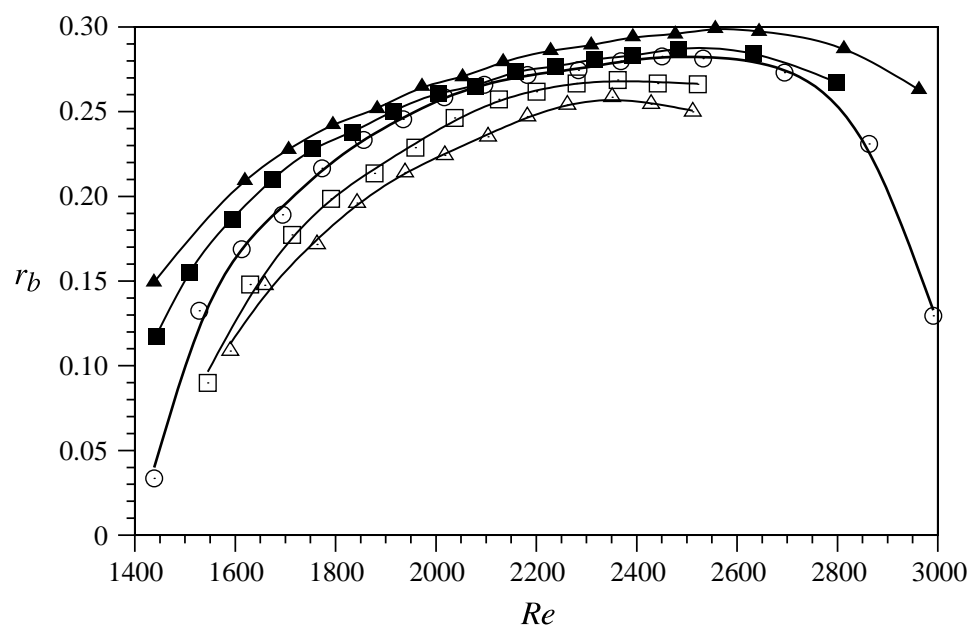

FIGURE 7. Variation of the normalized breakdown bubble radius with Reynolds number. Corotation (solid symbols) and counter-rotation (hollow symbols) cases are considered for the control disk $d_{3}$. Rotation rates as in figure 6. Symbols: circles, no control; squares, $\varepsilon= \pm 0.39$; triangles, $\varepsilon= \pm 1.23$.

for counter-rotation. It can be observed from these pictures that the axial and radial dimensions of the bottom bubble were roughly unchanged for the same rotation ratio, whether in co-rotation or counter-rotation.

\section{(b) Bubble radius}

The normalized radial size (percentage of $H$ ) of the vortex breakdown bubble is plotted against the rotating-endwall Reynolds number in figure 7. The results show the variation of the bubble radius with Reynolds number for the no-control, co-rotation $\left(R e_{d}=15\right.$ and 31$)$ and counter-rotation $\left(R e_{d}=15\right.$ and 31$)$ cases. The effects of corotating and counter-rotating the control disk on the radius of the main bubble are clearly shown. It is obvious that co-rotation of the control disk $d_{3}$ tended to increase the radial size of the breakdown bubble, while counter-rotation tended to decrease it. In addition, the effect of the control-disk rotation on the breakdown bubble was observed to be relatively more pronounced at lower Reynolds numbers and for those beyond the point where the bubble had reached it maximum size. After the bubble had reached its maximum size, further increases in the Reynolds number tended to have the opposite effect, similar to the no-control case.

\section{(c) Upstream stagnation point}

Figure 8 presents the movement of the upstream stagnation point for the main vortex breakdown bubble as the rotating-endwall Reynolds number increases. This movement is characterized by the normalized height of the stagnation point $\left(z_{u}\right)$. The cases for which the results are shown correspond to the no-control, co-rotation and counterrotation when the control-disk Reynolds number $\left(R e_{d}\right)$ was set to 31 . To put this in perspective, this Reynolds number corresponds to a rotation ratio $\varepsilon= \pm 0.92$ when the rotating-endwall Reynolds number is 1440 (this is at the onset of vortex breakdown for the no-control case). For the no-control case, the results show that the height of the upstream stagnation point decreases as the Reynolds number is increased. Eventually, $z_{u}$ reaches an apparent asymptotic level of $\sim 13 \%$ of the tank height $(2 R)$. The effect of co-rotation on the axial position of the upstream stagnation point was to shift it 


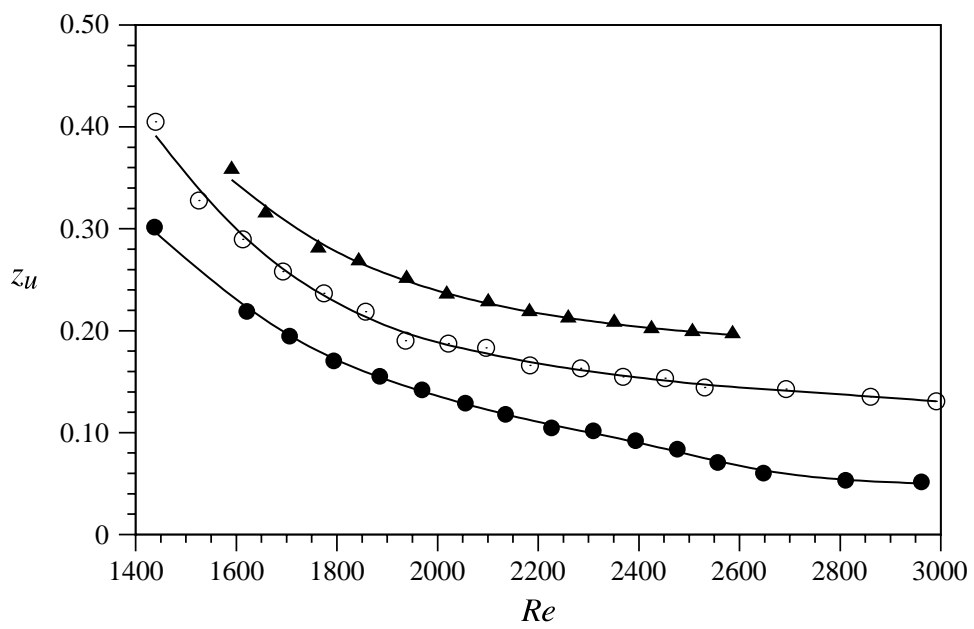

FIGURE 8. Variation of the normalized upstream stagnation point position with Reynolds number. Current results for no control (circles) are compared with the data of Fujimura, Koyama \& Hyun (1997) in figure 4. Co-rotation (circles) and counter-rotation (triangles) cases are considered for $d_{3}$ at $R e_{d}=31$.

further upstream, closer to the bottom endwall. On the other hand, counter-rotation tended to move the upstream stagnation point in the downstream direction, or closer to the rotating endwall. Other results, not reproduced here, showed that the higher the absolute magnitude of the rotation ratio, the more significant the shift from the no-control position.

\subsubsection{Effect of control-disk size}

The effect of the control-disk size on the vortex breakdown was investigated to quantify its effect on the location and size of the breakdown bubble. In these experiments, the rotating endwall was spun at constant angular velocity, while the rotation ratio was varied by changing the angular velocity of the control disks. Experimental results are presented as flow visualization pictures, and as plots of the normalized bubble radius and upstream stagnation height against the rotation ratio.

\section{(a) Flow visualization}

Co-rotation. Figures 9 and 10 show the effect of co-rotating control disks of different sizes $\left(d_{2}\right.$ and $\left.d_{4}\right)$ on the breakdown bubble size and location along the swirl axis. In both cases, the initial state inside the cylinder was that corresponding to the vortex breakdown onset as shown in figure $9(a)$. In figure $9(b-e)$, the control disk $d_{2}$ was gradually co-rotated with increasing rates to manipulate the onset breakdown bubble. The breakdown bubbles illustrated in figure $9(b-e)$ correspond to the rotation ratios $\varepsilon=0.45,1.38,2.32$ and 3.73 , respectively; those shown in figure $10(a-c)$ correspond to the control disk $d_{4}$ co-rotated at $\varepsilon=0.45,1.38$ and 2.32 , respectively.

It is important to note that, with the same co-rotation ratios $(\varepsilon=0.45,1.38$ and 2.32), the bubbles generated using the larger control disk, $d_{4}$ in this case, were significantly larger than those obtained using the smaller control disk, $d_{2}$. To be specific, the normalized radius of the breakdown bubbles enhanced using $d_{2}$ and $d_{4}$ yielded the following values: $0.06,0.10$ and 0.133 (for $d_{2}$ ) and $0.16,0.20$ and 0.21 
(a)

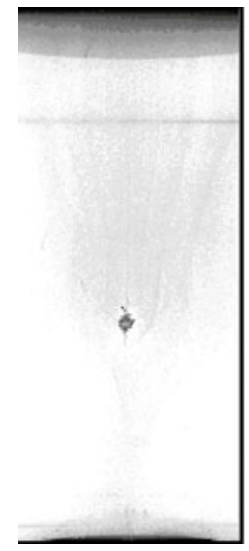

(b)

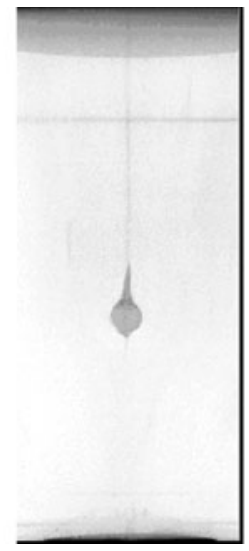

(c)

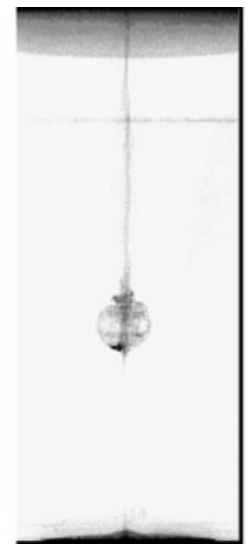

$(d)$

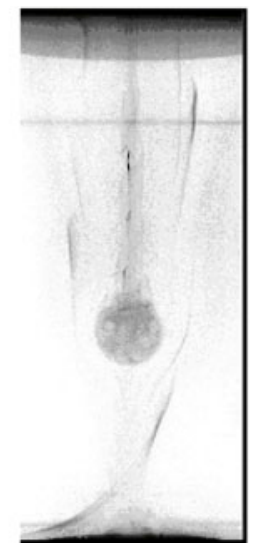

$(e)$

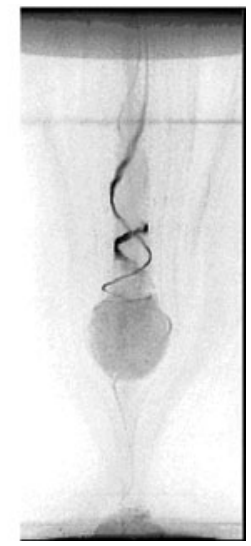

FIGURE 9. Flow visualization of vortex breakdown due to the rotation of the top endwall at $R e=1440$ and the effect of co-rotating the disk $d_{2}$ is depicted: $(a)$ no control; $(b) \varepsilon=0.45$; (c) $\varepsilon=1.38 ;(d) \varepsilon=2.32 ;(e) \varepsilon=3.73$.

(a)

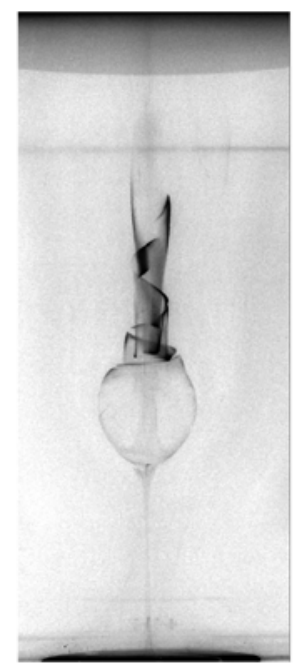

$(b)$

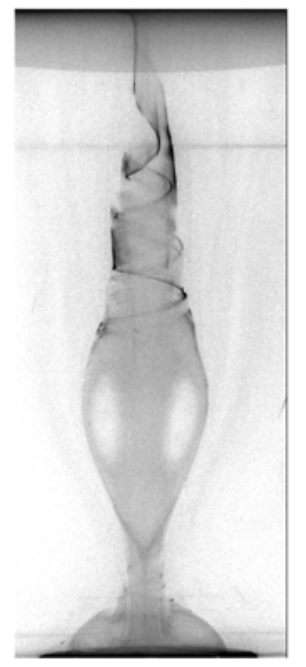

(c)

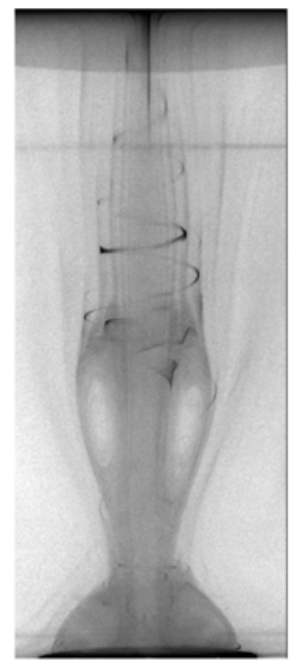

(d)

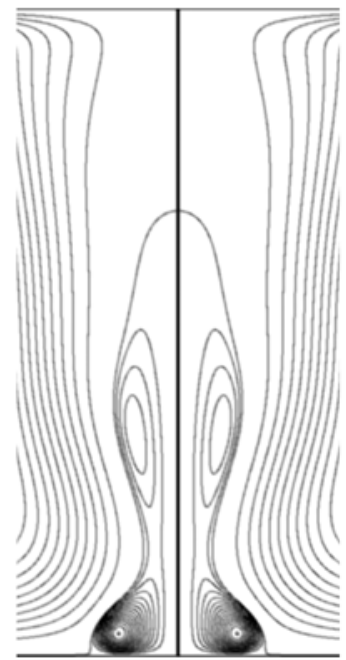

FIGURE 10. Flow visualization of vortex breakdown due to the rotation of the top endwall at $R e=1440$ and the effect of co-rotating the disk $d_{4}$ is depicted: $(a) \varepsilon=0.45 ;(b) \varepsilon=1.38$; (c) $\varepsilon=2.32$. These breakdown bubbles were generated from the initial vortex breakdown onset, as shown in figure $9(a)$. $(d)$ Streamlines from a numerical simulation (see $\S 4$ ) of the case shown in panel $(c)$ for comparison.

(for $d_{4}$ ). It is also clear from figures 8 and 9 that increasing rates of co-rotation shifted the bubble in the upstream direction.

The results depicted in figure 9 also indicate that the rate of increase of the breakdown bubble radius with co-rotation ratio is almost identical to the rate of increase of the axial size. In addition, the bubbles generated using $d_{4}$ were observed to be very sensitive to co-rotation, as the bubble radius tended to quickly reach a 
(a)

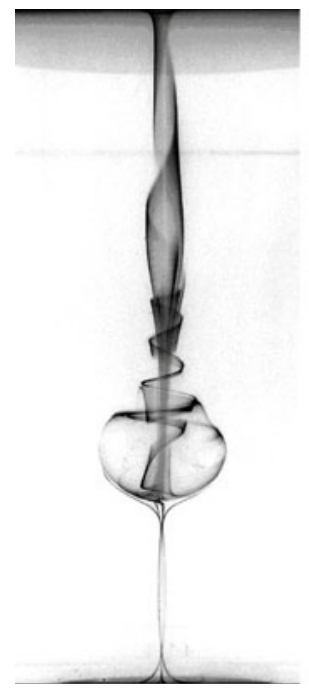

(b)

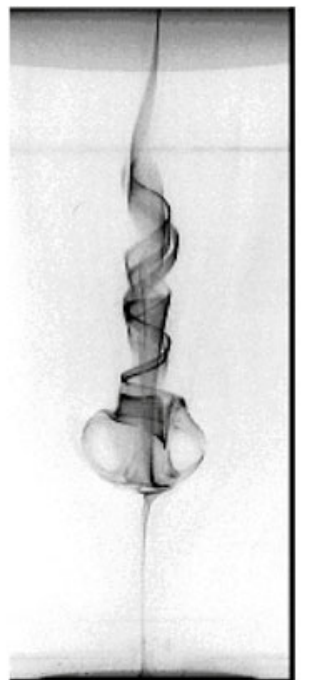

$(c)$

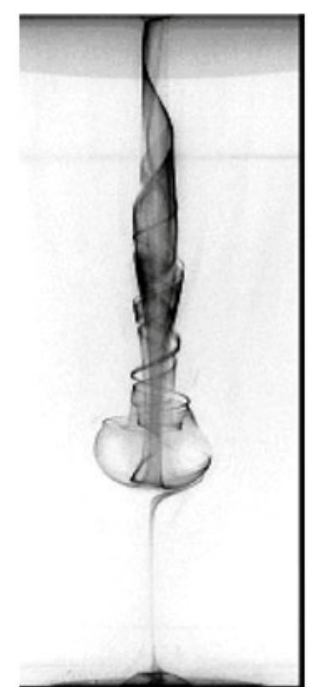

$(d)$

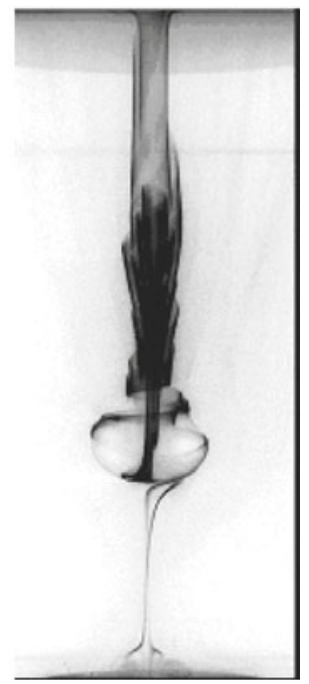

FIGURE 11. Flow visualization of vortex breakdown due to the rotation of the top endwall at $R e=1660$ and the effect of counter-rotating $d_{2}$ is depicted: (a) no control; $(b) \varepsilon=-0.36$; (c) $\varepsilon=-1.42 ;(d) \varepsilon=-2.84$.

size close to saturation (maximum size) as a result of only a minor rotation ratio (such as $\varepsilon=1.38$ ) followed by progressive stretching in the axial direction. While the normalized radial size of the breakdown bubble varied from 0.16 to 0.20 for a corresponding change in rotation ratio from 0.45 to 1.38 , a significant change in the normalized axial size was noted, from 0.21 to 0.38 . The growth of the bottom bubble, as observed with both control disks, was proportional to the rotation ratio. Figure $10(c)$ shows a flow structure where the main vortex breakdown is connected to the bottom bubble. In terms of downstream applications, this appears to result in a potentially very attractive flow structure conducive for cell or tissue growth, with a large laminar controlled region and associated low shear stress and significant residence time.

Counter-rotation. Figures 11 and 12 illustrate the effect of counter-rotating control disks of different dimensions on the breakdown bubble size and axial location. From the two sets of figures, although the initial flow structures as well as the rotation ratios were different, the results clearly show the benefit of using a larger control disk to suppress vortex breakdown. Figures 11(a) and 12(a) represent the initial breakdown states with no control and correspond to $R e=1660$ and 1920, respectively. A higher starting Reynolds number for control disk $d_{4}$ was used to be able to sustain the breakdown state in the presence of slight counter-rotation. This clearly indicates how strong the effect of counter-rotating a disk the size of $d_{4}$ is on the breakdown bubble. (The area of disk $d_{4}$ is still only $\sim 4 \%$ of the endwall disk area.) The breakdown conditions depicted in figure $11(b-d)$ correspond to the control disk $d_{2}$ counter-rotated at $\varepsilon=-0.36,-1.42$ and -2.84 , respectively. The breakdown bubbles shown in figure $12(b, c)$ correspond to the control disk $d_{4}$ counter-rotated at $\varepsilon=-0.33$ and -0.56 , respectively.

A close analysis of figure $11(a-d)$ reveals that the smaller control disk $d_{2}$ changed the structure of the vortex breakdown only very marginally, this being true even with a higher value of the counter-rotation ratio, $\varepsilon=-2.84$. In contrast, the larger control 
(a)

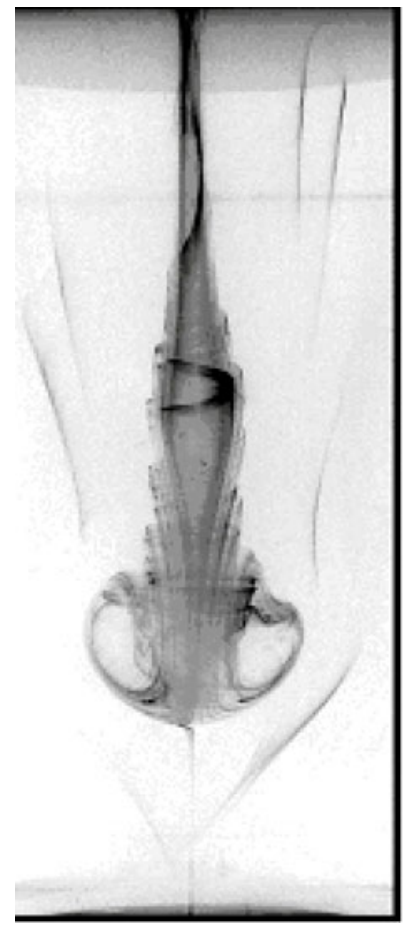

(b)

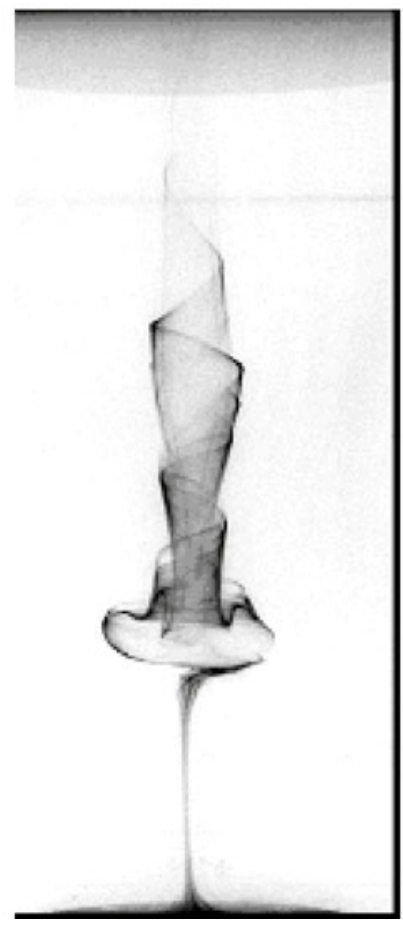

$(c)$

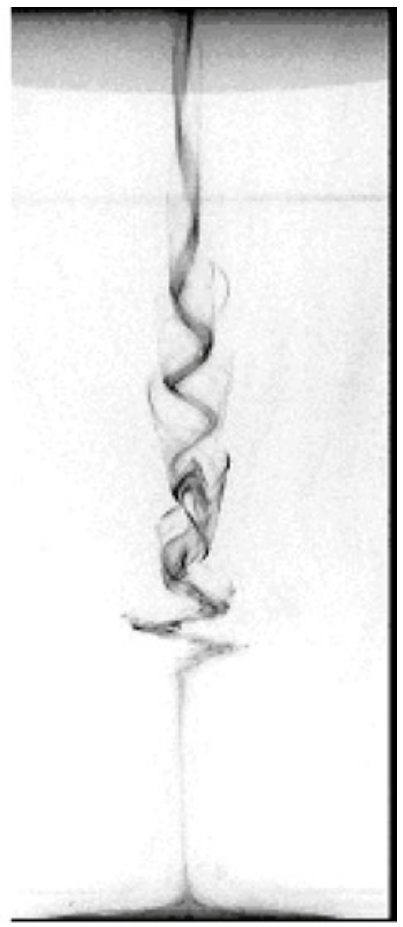

FIGURE 12. Flow visualization of vortex breakdown due to the rotation of the top endwall at $R e=1920$ and the effect of counter-rotating $d_{4}$ is depicted: $(a)$ no control; $(b) \varepsilon=-0.33$; (c) $\varepsilon=-0.56$.

disk $d_{4}$ was capable of completely suppressing the larger initial vortex breakdown bubble depicted in figure $12(a)$ at a relatively slower counter-rotation ratio, $\varepsilon=-0.56$. The general tendency observed in both cases (i.e. counter-rotation with $d_{2}$ and $d_{4}$ ) was that the breakdown bubble appeared to be compressed in the radial direction and more so in the axial direction, while the size of the downstream vortex core appeared to increase. In particular, a counter-rotation ratio of $\varepsilon=-0.33$ using the control disk $d_{4}$ caused a reduction in the normalized axial size of the bubble from 0.19 to 0.095 , whereas the compression due to $d_{2}$ was insignificant for a similar rotation ratio. The change in the radius was again more marked for $d_{4}$ than for $d_{2}$. The results also showed that, for higher counter-rotation ratio, a clearly observable bottom bubble was generated.

The next set of results (figures 13 and 14) show the effect of the control-disk size on the normalized radius and height of the upstream stagnation point of the breakdown bubble. This time the rotating endwall was spun with a constant angular velocity: in co-rotation at $R e=1440$ and in counter-rotation at $R e=1660$. In experiments involving co-rotation, the spinning of the control disks was the catalyst for vortex breakdown formation, from the initial onset state, shown in figure $9(a)$, to increasing growth. For the counter-rotation cases, the initial flow structure inside the cylinder was characterized by the presence of a large bubble, as depicted in figure 11 $(a)$. Gradual increases of the counter-rotation rate caused the breakdown bubble to shrink. 


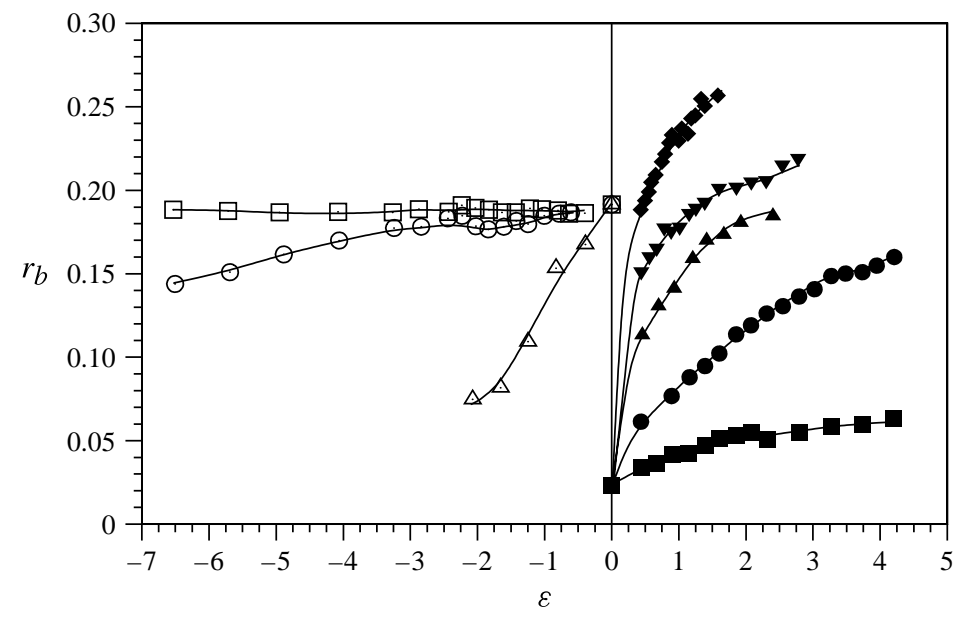

FIGURE 13. Variation of the breakdown bubble radius with rotation ratio. The effects of five control disks, $d_{1}-d_{5}$, are compared. Co-rotation (solid symbols) starts with $R e=1440$ while counter-rotation (hollow symbols) starts with $R e=1660$. Symbols: $d_{1}$, squares; $d_{2}$, circles; $d_{3}$, triangles; $d_{4}$, inverted triangles; $d_{5}$, diamonds).

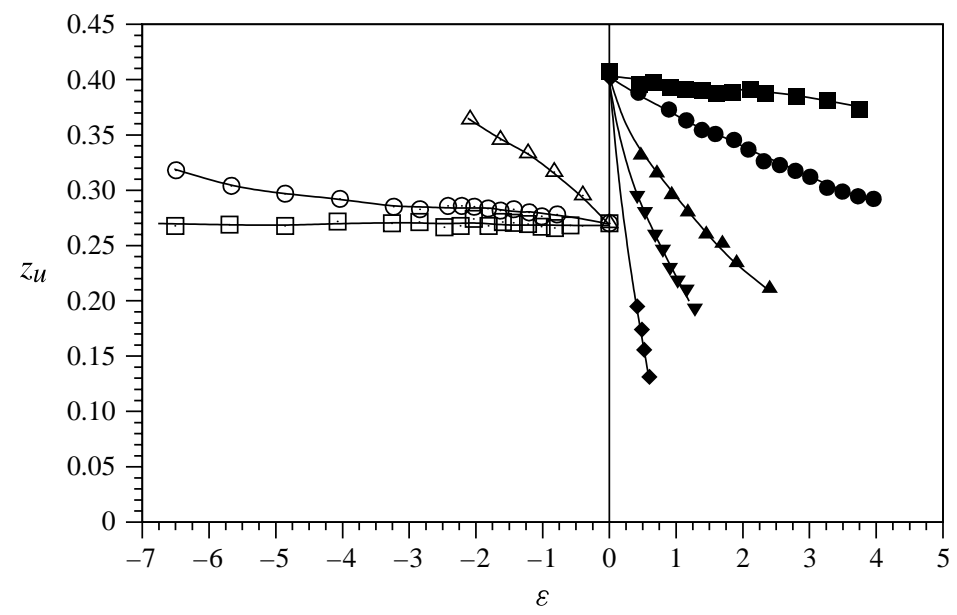

FIGURE 14. Variation of the breakdown bubble upstream stagnation point with rotation ratio. The effects of five control disks, $d_{1}-d_{5}$, are compared. Co-rotation (solid symbols) starts with $R e=1440$ while counter-rotation (hollow symbols) starts with $R e=1660$. Symbols: $d_{1}$, squares; $d_{2}$, circles; $d_{3}$, triangles; $d_{4}$, inverted triangles; $d_{5}$, diamonds).

\section{(b) Bubble radius for co- and counter-rotation}

Co-rotation of the control disk with the base flow initially at the onset state was performed with all the five control disks. Looking at the results in figure 13, it is clear that the size of the control disk has a significant effect on the size of the breakdown bubble. To demonstrate the effect of the different disks in co-rotation on the onset breakdown bubble, we consider all control disks rotating such that $\varepsilon \approx 2.40$. As a result, control disks $d_{1}, d_{2}, d_{3}$ and $d_{4}$ caused an increase in the radial size of the bubble in the following order: 2.0, 5.0, 7.2, and 8.0, respectively. The control disk $d_{5}$ 
caused a bubble radius increase by a factor of 10 when co-rotating at a relatively lower rotation ratio, $\varepsilon=1.60$. Again, these results clearly show that the size of the control disk has the most significant bearing on the size of the breakdown bubble generated by co-rotation.

In figure 13, the normalized breakdown bubble radius is plotted against the rotation ratio, and the effect of co-rotating and counter-rotating control disks of different sizes is presented. The smallest disk $d_{1}$ appeared to have no noticeable effect on the size of the breakdown bubble even at a very high counter-rotation ratio of $\varepsilon=-6.50$. While counter-rotation of control disk $d_{2}$ appeared to cause some degree of shrinkage to the breakdown bubble, it is obvious that $d_{3}$ had the largest reduction effect on the bubble radius. To illustrate this, for a counter-rotation ratio of approximately -1.60 , the control disk $d_{2}$ caused the bubble radius to shrink to only $92 \%$ of the original bubble size, corresponding to $R e=1660$ with no control, whereas $d_{3}$ caused a corresponding shrinkage to $42 \%$.

\section{(c) Upstream stagnation point for co- and counter-rotation}

Figure 14 presents the variation of the normalized upstream stagnation point position with changes of rotation ratio using the five experimental small disks. The results show that, in counter-rotation, the smallest control disk $d_{1}$ only negligibly affected the axial location of the bubble, as illustrated by an almost horizontal line in the graph. Counter-rotation using $d_{2}$ and $d_{3}$ revealed a downstream shift of the upstream stagnation point, with $d_{3}$ exhibiting the larger movement. However, for co-rotation, the general trend for all control disks was an upstream movement of the stagnation point. Again, the control disk $d_{1}$ showed the least effect as opposed to the largest disk $\left(d_{5}\right)$, which only needed a slight rotation ratio $\varepsilon=0.61$ to move the breakdown bubble from a normalized height of 0.40 to 0.13 . Such a shift is by all means highly significant considering the slight co-rotation ratio employed. On the other hand, the control disk $d_{1}$ co-rotating with a much higher rotation ratio $\varepsilon=4.2$ caused only a marginal shift of the upstream stagnation point from $\sim 0.40$ to only $\sim 0.36$.

\section{Further analysis}

To obtain further insight into the mathematical/physical mechanism delaying or enhancing vortex breakdown through the use of a small control disk, numerical simulations were undertaken to provide details of the flow and vorticity fields, especially in the neighbourhood of the disk. The incompressible axisymmetric Navier-Stokes equations were solved using a spectral-element technique (e.g. Karniadakis \& Sherwin 1999). The simulations were undertaken on a 1250 macroelement mesh with mesh compression towards the no-slip boundaries where the gradients are very large. The mesh was also refined locally at the outer radius of the small disk, where there is an azimuthal velocity discontinuity. The geometry was split into quadrilateral elements, and, within these elements, the velocity and pressure fields were represented by third-order tensor-product Lagrange polynomials, based on Gauss-Lobatto-Legendre quadrature points. Switching to fifth-order polynomial elements resulted in a change to the critical Reynolds number for the onset of breakdown from $R e_{c}=1445.2$ to $R e_{c}=1444.6$, a difference of less than $0.05 \%$. The critical Reynolds number was obtained by incrementing the Reynolds number in small increments $(\Delta R e=10)$ and then using linear interpolation to predict when the minimum axial velocity first became zero. Then further simulations were performed close to the predicted value, again using linear interpolation to refine the result. The predicted shift in the critical Reynolds number as a function of small-disk rotation 


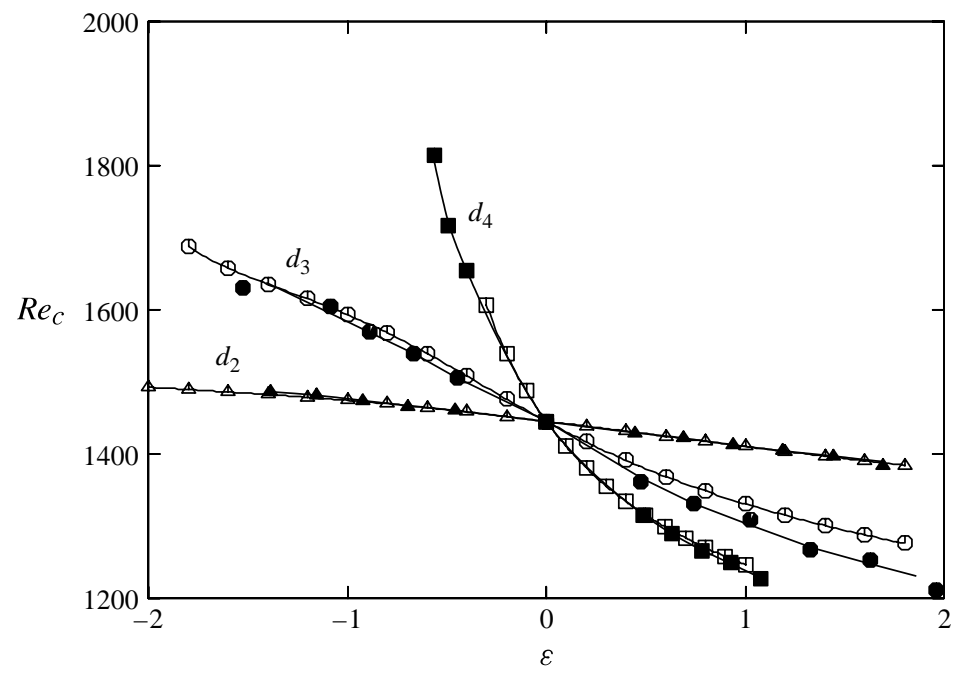

FIGURE 15. Comparison of numerical predictions and experimental findings for the shift in the critical Reynolds number for breakdown onset with rotation ratio and disk size. The filled symbols show experimental results and the open symbols numerical results. The disk size ratio is marked.

ratio and size (open symbols) is shown in figure 15, with the experimental results (solid symbols) overlaid. The clearly two sets of results match well, although there is a slight mismatch for positive rotation rates for disk $d_{3}$. In addition, a visual comparison of experimental and numerical results for one of the extreme breakdown cases is shown in figure $10(c, d)$. Details of this approach and implementation can be found in Thompson, Hourigan \& Sheridan (1996) and Thompson et al. (2006), and it has been employed successfully on a variety of axisymmetric problems (e.g. Thompson \& Hourigan 2003; Leweke, Thompson \& Hourigan 2004; Mununga et al. 2004; Thompson, Leweke \& Hourigan 2007; Griffith et al. 2009).

\subsection{Vorticity generation}

Since there is no dependence on the azimuthal coordinate, it is possible to construct a two-dimensional (Stokes) streamfunction, $\Psi(z, r)$, which defines the velocity components $\left(u_{z}=(1 / r) \partial \Psi / \partial r, u_{r}=-(1 / r) \partial \Psi / \partial z\right)$ in an azimuthal plane. Therefore, the flow state can be defined in terms of the streamfunction, which is forced by the azimuthal vorticity, $\omega_{\theta}$, and governed by the equation

$$
\frac{\partial}{\partial r} \frac{1}{r} \frac{\partial \Psi}{\partial r}+\frac{\partial}{\partial z} \frac{1}{r} \frac{\partial \Psi}{\partial z}=-\omega_{\theta},
$$

together with the azimuthal velocity component $u_{\theta}$ and the azimuthal vorticity. Thus it is clear that the azimuthal vorticity component plays an important role in whether vortex breakdown can occur, as has been pointed out by a number of authors (e.g. Brown \& Lopez 1990). Physically, the addition of a vortex ring with negative (anticlockwise in the following figures) vorticity at the position where a breakdown bubble may occur will lead to a reduction in the upwards velocity on the cylinder axis, through Biot-Savart induction, thus encouraging the flow to reverse direction. Moreover, generation of increased negative (or positive) azimuthal vorticity in the 
neighbourhood of a breakdown region will enhance (or retard) the development of the breakdown. This is relevant to the cases examined here, as discussed below.

The equation governing the evolution of azimuthal vorticity can be written in cylindrical coordinates as

$$
\frac{\mathrm{D} \omega_{\theta}}{\mathrm{D} t}=\frac{\omega_{\theta} u_{r}}{r}+\frac{\partial}{\partial z}\left(\frac{u_{\theta}^{2}}{r}\right)+\frac{1}{R e}\left[\frac{\partial}{\partial r}\left(\frac{1}{r} \frac{\partial r \omega_{\theta}}{\partial r}\right)+\frac{\partial^{2} \omega_{\theta}}{\partial z^{2}}\right] .
$$

The term on the left-hand side is the rate of change of vorticity of a fluid element moving with the fluid. The first term on the right-hand side can be thought of physically as the change in vorticity induced by stretching as an axial vortex ring increases in radius. (As the ring circumference increases, the cross-sectional diameter decreases to conserve volume, leading the peak vorticity to increase so that circulation is conserved.) The second term represents a source of azimuthal vorticity dependent on the azimuthal velocity component. This is a differential centrifugal pumping term. Consider a cylindrical shell of fluid at a fixed radius with the swirl velocity increasing with axial distance. In this case the centrifugal force will tend to cause fluid swirling faster to move outwards radially relative to more slowly swirling fluid. In turn, this generates a velocity gradient $\partial u_{r} / \partial z$, and hence generates positive azimuthal vorticity (since $\omega_{\theta}=\partial u_{r} / \partial z-\partial u_{z} / \partial r$ ). This term will be denoted $S_{\theta}$ as it plays a central role in the controlled configuration. The other terms are radial and axial viscous diffusion terms, which only slowly redistribute vorticity, without changing the circulation.

\subsubsection{Observations and predictions from numerical simulations}

Figure 16 shows the streamlines at $R e=1450$, which is marginally above the critical Reynolds number for breakdown $(R e=1445.2)$. The azimuthal vorticity distribution is also shown for comparison. The overlaid solid contour effectively marks the position of a vortex ring, which induces downwards flow along the axis through Biot-Savart induction. Note that, not coincidentally, the centre of this effective vortex ring, shown by the ' + ', is approximately at the axial position at which the axial velocity reverses sign. Also shown is the vorticity source distribution, which feeds the vortex ring with anticlockwise vorticity as fluid advects through it along the streamline indicated by the dashed line.

The effect of the small control disk is to modify the vorticity source upstream of the vortex ring, which controls the induced axial backflow, and, in turn, the size of the vortex breakdown bubble or its absence. To investigate this further, simulations were performed with the intermediate control disks $\left(d_{2}, d_{3}\right.$ and $\left.d_{4}\right)$, for different rotation rates $(\varepsilon=0, \pm 0.1, \pm 0.2, \pm 0.3, \pm 0.4, \pm 0.5)$. The incremental azimuthal vorticity source distribution relative to the uncontrolled case was then calculated for each case, i.e. $\Delta S_{\theta}=S_{\theta}(\varepsilon)-S_{\theta}(\varepsilon=0)$.

Figure 17 shows the incremental source distribution for control-disk rotation rates $\varepsilon= \pm 0.1, \pm 0.5$. The contour level ranges have been scaled by $\varepsilon$ to show that, at least away from the bottom left-hand corner, the incremental source distribution is almost spatially identical when scaled (linearly) by $\varepsilon$. At the bottom left-hand corner, the effect of the control disk is to centrifuge fluid outwards radially, effectively independent of the rotation direction. However, away from that region, the net effect appears to be the proportional injection of positive or negative swirl into the fluid, which, in turn, has almost a linear effect on $\Delta S_{\theta}$. Notice that the streamline passing through the vortex ring, discussed above, passes well above the region where the scaled incremental vorticity source changes nonlinearly with rotation rate. 
(a)

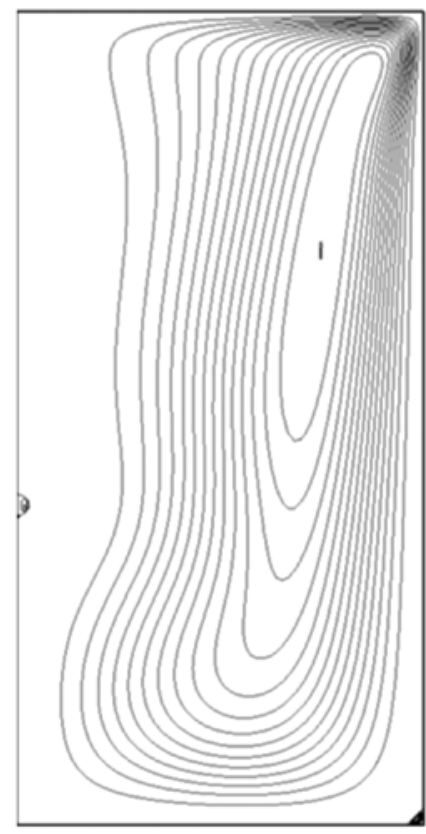

(b)

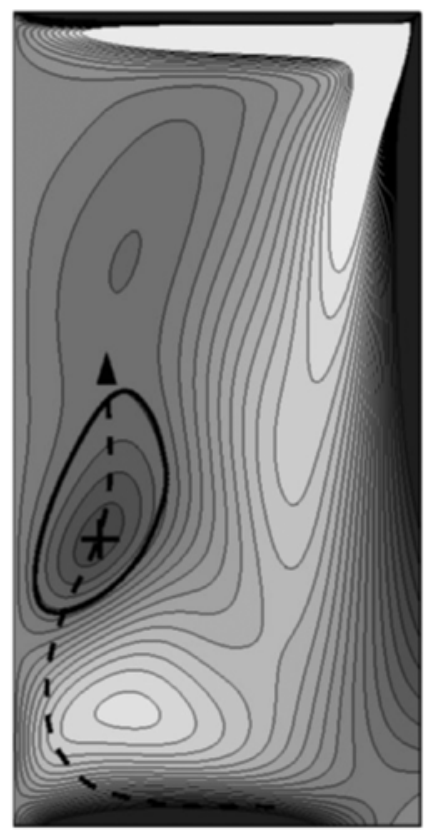

(c)

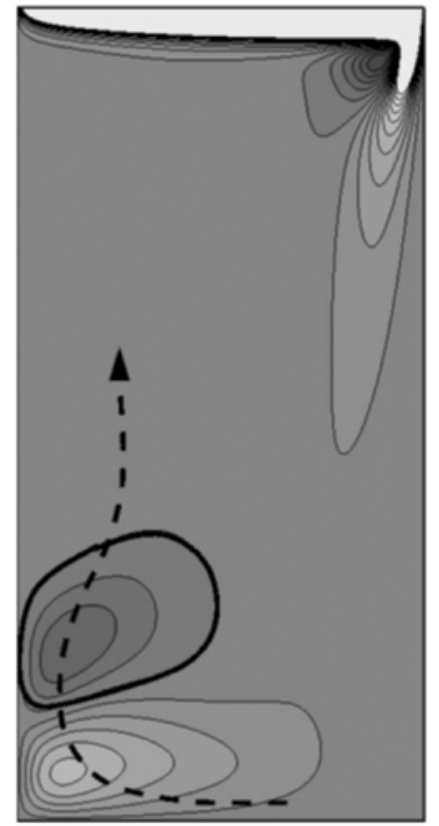

FIgURE 16. (a) Streamlines in the meridional plane for $R e=1450$ just above the onset of breakdown. (b) Greyscale plot of the azimuthal vorticity field; anticlockwise rotation corresponds to dark grey and clockwise rotation is shown as light grey. The overlaid solid line with the cross at the centre highlights what can effectively be considered as (a cross-section through) a vortex ring, which induces downward flow along the axis (due to Biot-Savart induction) resulting in vortex breakdown. (c) Greyscale plot of the vorticity source term $S_{\theta}$, which feeds anticlockwise vorticity into the azimuthal vortex ring as fluid advects along a streamline shown by the dashed line.

Figure 18 shows a plot of the incremental source term, $\Delta S_{\theta} / \varepsilon$, moving along the streamline shown in figure 16 for the same four rotation ratios depicted in figure 17. Clearly, there is very little difference in the scaled incremental source for these cases. Also shown is the time integral of the incremental source term, again moving along the same streamline. Again, there is only a minor difference in the integrated source evolution due to quite different control-disk rotation rates, and in particular the curves show very similar values at the axial position close to where breakdown occurs. This quantity is a direct measure of the induced change to the azimuthal vorticity along this streamline, caused by the additional source/sink generated by the small-disk rotation.

Thus, directly from Biot-Savart induction from this added/reduced vorticity, the incremental change to the axial velocity induced by the control disk, near the location of breakdown, will also scale with $\varepsilon$. In addition, the incremental source could be expected to vary with disk diameter. Figure 19 plots the scaled change to the axial velocity along the centreline for 10 different rotation rates for the three intermediate disk sizes. The dependent variable is $\Delta u_{z}^{\prime}=\left(u_{z}-u_{z, \varepsilon=0}\right) /(\varepsilon d)^{3}$. This indicates that the incremental change to the axial velocity induced by the control disk for an axial range of $0.3<z<0.4$ is given approximately by the linear relationship

$$
\Delta u_{z}^{\prime}=[(-4.5 \pm 1.5)+60(z-0.35)] \varepsilon d^{3} .
$$


(a)

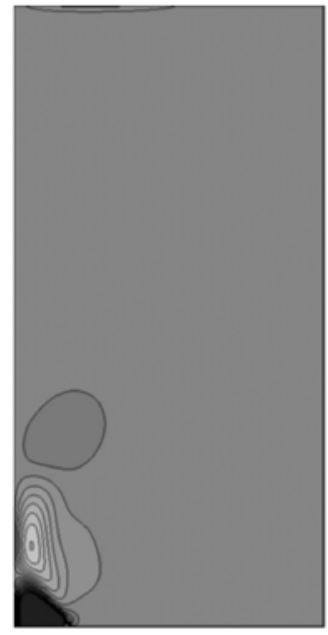

(b)

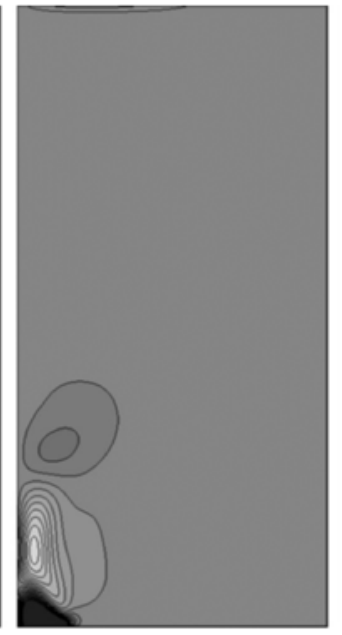

(c)

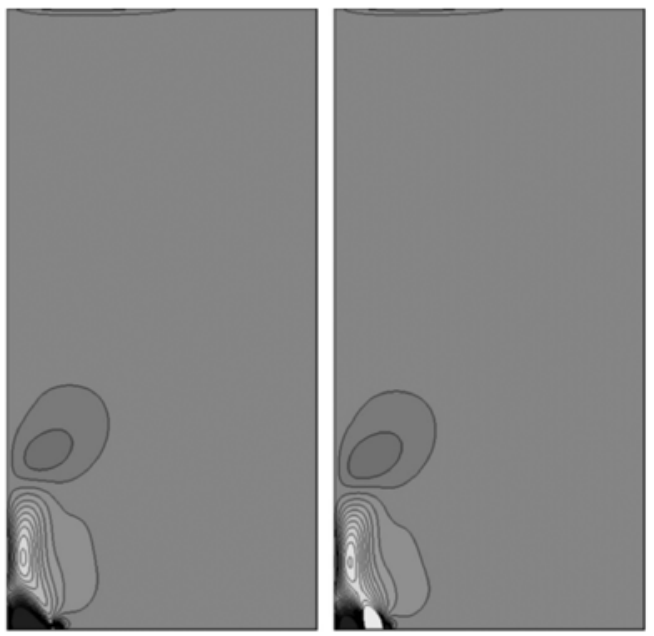

FIGURE 17. $(a-d)$ Incremental vorticity source distributions for $\varepsilon=-0.5, \quad-0.1,0.1$ and 0.5 , respectively. For these plots, the contour levels cover the ranges $-0.1 \leqslant S_{\theta} \leqslant 0.1$, $-0.02 \leqslant S_{\theta} \leqslant 0.02,0.02 \geqslant S_{\theta} \geqslant-0.02$ and $0.1 \geqslant S_{\theta} \geqslant-0.1$, respectively, i.e. they cover the same range when scaled by $\varepsilon$. Thus, the effect of the spinning control disk on the incremental vorticity source distribution is almost linear with rotation speed, at least away from the bottom left-hand corner. These contour plots correspond to disk $d_{3}$.

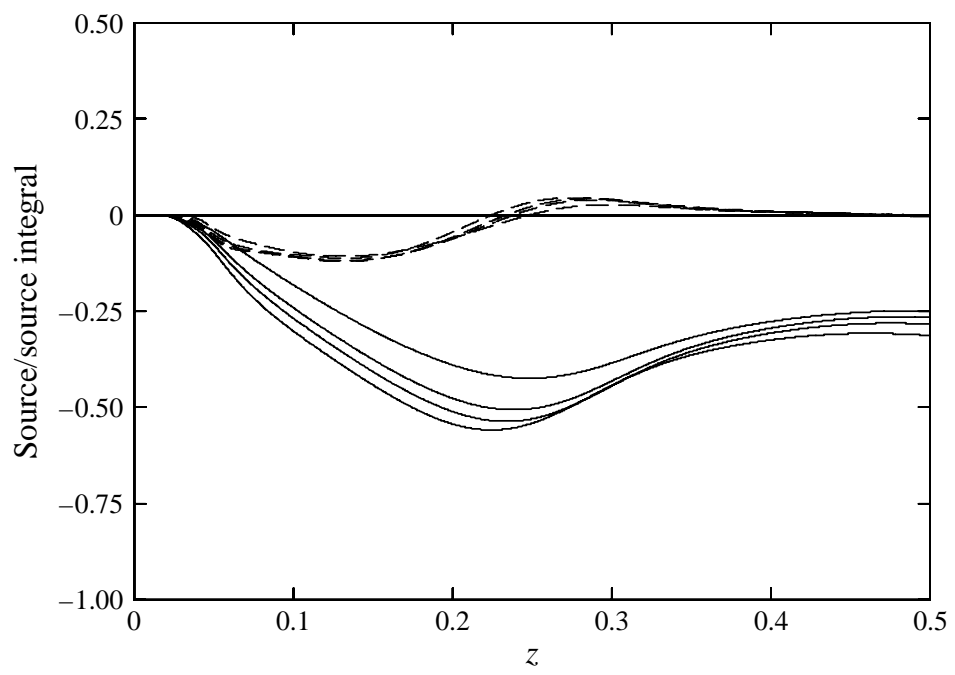

FIGURE 18. The dashed lines show the scaled incremental vorticity source, $\Delta S_{\theta} / \varepsilon$, along the streamline shown in figure 16 for the disk $d_{3}$ for $\varepsilon= \pm 0.1, \pm 0.5$ (as for figure 17). The solid lines show the time integral of the source moving along the streamline. Despite a large variation in the rotation rate, the integrated source (which can be associated with an azimuthal vorticity change to the main flow) remains semi-quantitatively similar, especially near the axial position at which breakdown first occurs. 


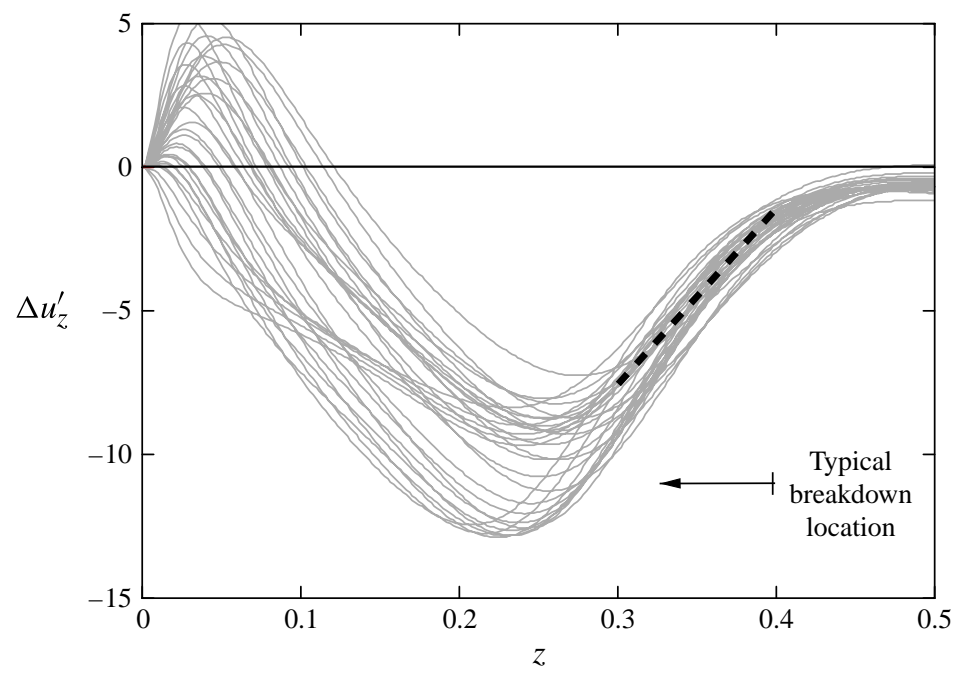

FIGURE 19. Scaled axial velocity increment against axial distance from the control disk for 10 different rotation rates $\varepsilon= \pm 0.1, \pm 0.2, \pm 0.3, \pm 0.4$ and \pm 0.5 , and three different disk sizes $d_{2}, d_{3}$ and $d_{4}$, showing a reasonable collapse of the curves near the axial position of vortex breakdown. A linear fit over this range is shown by the thick dashed line.

This applies to a relatively wide range of rotation rates and disk sizes. (Note that at onset breakdown occurs at $z_{u} \simeq 0.4$, and this reduces as the breakdown bubble increases in size. Also note that the variables are dimensionless, i.e. $z$ is the axial height scaled by the rig height $(H), d$ is the ratio of the small to large disk diameters, and the axial velocity increment is scaled by the endwall disk velocity at its outer radius, prior to then further scaling by $\varepsilon / d^{3}$.)

Furthermore, it seems likely that this same correction to the axial velocity on the centreline will still apply approximately over a range of Reynolds numbers near the critical Reynolds number as the base flow changes, i.e. the incremental source will only depend weakly on the base flow. The variation of the minimum axial velocity with Reynolds number for the uncontrolled case, determined from direct simulations without the control disk, is shown in figure 20(a). In addition, the axial position at which this minimum occurs is plotted in figure $20(b)$. Linear fits about the breakdown Reynolds number $\left(R e_{c}=1445.2\right)$ are overlaid. These fits are given by

$$
\begin{gathered}
z_{\min }=0.396-0.000575\left(R e-R e_{c}\right), \\
u_{z_{\min }}=-0.0000539\left(R e-R e_{c}\right) .
\end{gathered}
$$

Using these two expressions together with the approximation for $\Delta u_{z}^{\prime}$, given by (4.3) above, gives to first order in $\varepsilon d^{3}$ an approximation for the variation of the critical Reynolds number as a function of $\varepsilon$ and $d$ :

$$
\Delta R e_{c} \simeq-32200 \varepsilon d^{3} \text {. }
$$

This variation is shown in figure 21 by the solid line. The experimental data for disks $d_{2}$ and $d_{3}$ (from figure 5) is shown for comparison. (The data for disk $d_{4}$ are not shown since even small rotation ratios induce large changes to the critical Reynolds number.) First, the experimental data collapse reasonably well according to the scaling 

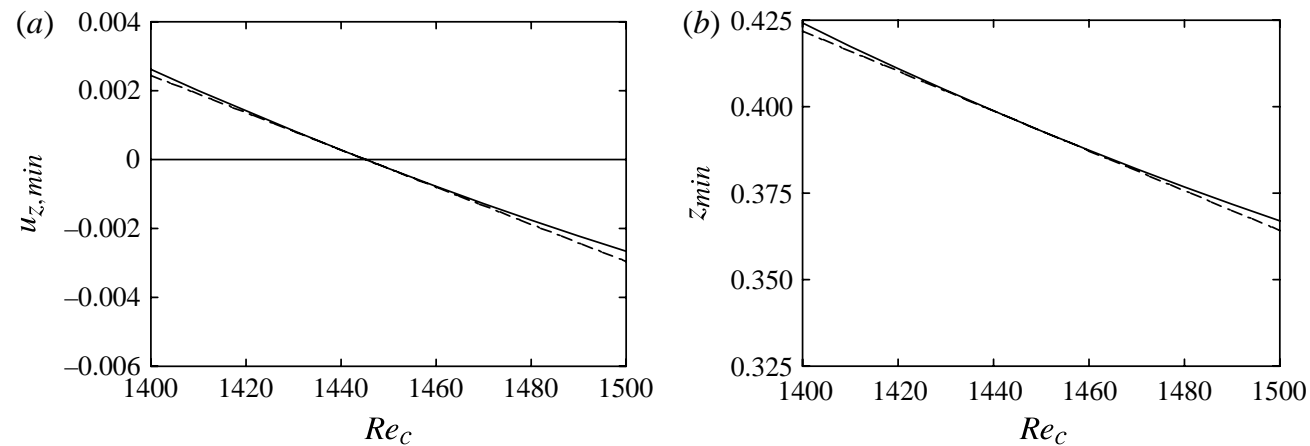

FIGURE 20. (a) Variation of the minimum axial velocity on the centreline with Reynolds number obtained from simulations without the control disk. (b) Axial position at which this minimum occurs. Linear fits to these variations are shown by the dashed lines.

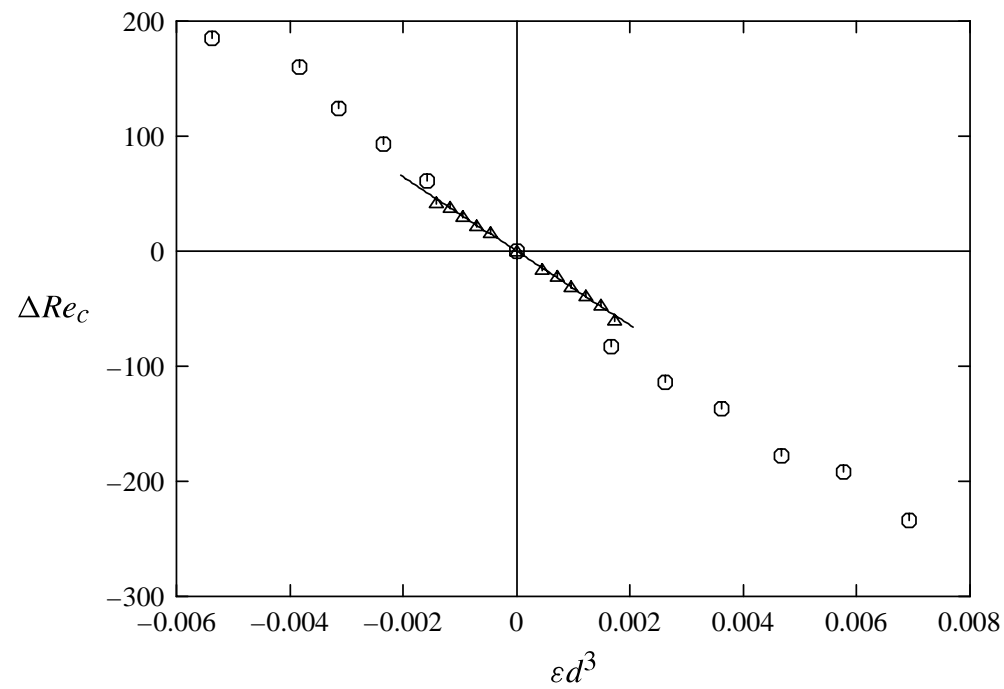

FIGURE 21. Predicted variation in the critical Reynolds number with $\varepsilon d^{3}$. The experimental values are shown by the triangles $\left(d_{2}\right)$ and circles $\left(d_{3}\right)$.

relationship. Second, the observed experimental variation is predicted remarkably well by (4.6), especially for small values of $\varepsilon d^{3}$.

In summary, the rotation of the small disk acts directly to increase/reduce the magnitude of the swirl velocity of the fluid above it for co-/counter-rotation, relative to the background swirl generated by rotation of the large disk alone. There is a strong but local nonlinear effect close to the disk, which varies considerably with control-disk rotation rate and disk size; however, further downstream, the incremental azimuthal vorticity source term distribution, $\Delta S_{\theta}$, scales extremely well with $\varepsilon d^{3}$. Since the vortex ring associated with breakdown, seen in figure $16(b)$, is fed by fluid moving along streamlines which avoid the variable source near the control disk but pass through the invariant source distribution further downstream, the net effect of the control disk also scales approximately with $\varepsilon d^{3}$. A local fit to the control-disk- 
induced scaled incremental axial velocity change is approximately linear with axial position. This can be used to approximately predict the shift in the critical Reynolds number with control-disk size and rotation rate, which is shown to be valid for small perturbations. Thus, in essence, there is an almost linear effect of the control disk with $\varepsilon d^{3}$ on the velocity distribution in the neighbourhood of the breakdown region, even though the overall effect on the flow is somewhat more complicated.

\section{Conclusions}

A non-intrusive method of controlling vortex breakdown, based on co-rotation and counter-rotation of a small disk embedded in the non-rotating endwall, has been presented and evaluated. For the no-control case, the experimental approach was validated against the breakdown observations and stability chart of Escudier (1984), and more quantitatively against breakdown location results from Fujimura et al. (2004). Results quantifying the onset of vortex breakdown with the control-disk rotation have shown that it occurs at lower Reynolds numbers for co-rotation, whereas it can be delayed for counter-rotation. Thus, this study has shown that co-rotation and counterrotation of a small control disk are efficient means to enhance or suppress vortex breakdown. Interestingly, this is opposite to the behaviour observed with a small rotating rod, where co-rotation suppresses breakdown and counter-rotation enhances it (Herrada \& Shtern 2003b; Husain et al. 2003; Lo Jacono et al. 2008). That behaviour can be interpreted in terms of downstream swirl decay considered by Shtern et al. (2012). The co-rotating rod (disk) decreases (increases) swirl decay downstream. In turn, the suction by pressure difference is weakened (strengthened) by a co-rotating rod (disk). As a result of suction strengthening (weakening), the flow reversal (i.e. vortex breakdown) occurs at smaller (larger) Reynolds number with the co- (counter-) rotating small disk than that with no control.

The findings have also revealed that, for the case of an existing finite-sized breakdown bubble, its size can be augmented by co-rotating the control disk. By contrast, counter-rotation caused the bubble size to shrink. The effect on the size of the breakdown bubble is amplified considerably as the size of the control disk is increased. In addition, co-rotation tended to push the breakdown bubble in the upstream direction, away from the rotating endwall, while counter-rotation caused the bubble to migrate further downstream. Again, it was observed that larger control disks produced a much more than linear shift in position, as was also observed for the bubble size. However, the amount of shift in the breakdown bubble position was approximately proportional to the absolute value of the rotation ratio.

From a practical point of view, the experiments have identified that it is beneficial to use a disk of a certain radius (e.g. size $\sim d_{4}$ corresponding to $4 \%$ of the endwall area), which gives a strong effect but still allows sensitive tuning of the influence on the bubble. Larger disks (e.g. $d_{5}$ ) do not allow the same fine tuning of breakdown bubble characteristics, while smaller disks do not provide a large enough effect.

Apart from the experimental results, numerical predictions were obtained using a spectral-element method for Reynolds numbers close to the critical value for a range of control-disk sizes and rotation rates. The purpose of these calculations was mainly to gain a greater insight into the underlying physical mechanisms responsible for flow control, rather than to reproduce the experimental results. Indeed, by providing details of the flow fields as a function of control parameters, it is clear that rotation of the control disk proportionally changes the azimuthal vorticity source upstream of breakdown, which subsequently increases or decreases the azimuthal vorticity in 
the neighbourhood of the breakdown location, thus modifying the induced axial flow along the axis through Biot-Savart induction. The modification to the axial velocity is shown to vary approximately with $\varepsilon d^{3}$. This can be combined with the measured change in axial velocity with Reynolds number for the uncontrolled case obtained from direct simulations to predict the experimentally observed shift in the critical Reynolds number with rotation rate and disk size.

This non-intrusive method has proved to be a very effective way of controlling vortex breakdown in an enclosed cylindrical vessel. The success of this method provides hope that it can be adapted to real-life applications to reduce or completely suppress vortex breakdown when it is detrimental or to enhance it when it is beneficial to the process of interest. More generally, it shows that small manipulation of the swirl immediately upstream of the bubble (with minimal energy input) is an effective means of control. An area that may benefit from this approach is in bioreactor design, where a controlled flow environment with low shear stress levels can be beneficial for growing certain types of cells. Such a controlled environment can be achieved by corotating a control disk, with a size and rotation ratio dictated by specific requirements. Cell growth experiments based on some of these ideas are under development within the Division of Biological Engineering at Monash University.

\section{Acknowledgement}

This research was supported by Australian Research Council (ARC) Discovery Grant DP0452664.

\section{REFERENCES}

Benjamin, T. B. 1962 Theory of the vortex breakdown phenomenon. J. Fluid Mech. 14, 593-629.

BhatTACHARYya, S. \& PAL, A. 1998 Axisymmetric vortex breakdown in a filled cylinder. Intl J. Engng Sci. 36, 555-563.

BhatTACHARYyA, S. \& PAL, A. 1999 Generation (or degeneration) of a separation bubble in a liquid-filled cylinder through spin-up (or spin-down) process. Trans. ASME: J. Appl. Mech. 66 (4), 1023-1026.

Billant, P., Chomaz, J. M. \& Huerre, P. 1998 Experimental study of vortex breakdown in swirling jets. J. Fluid Mech. 376, 183-219.

Brøns, M., Shen, W. Z. \& SøRensen, J. N. 2007 The influence of imperfections on the flow structure of steady vortex breakdown bubbles. J. Fluid Mech. 578, 453-466.

Brøns, M., Thompson, M. C. \& Hourigan, K. 2009 Dye visualization near a three-dimensional stagnation point: application to the vortex breakdown bubble. J. Fluid Mech. 622, 177-194.

Brown, G. L. \& Lopez, J. M. 1990 Axisymmetric vortex breakdown. Part 2. Physical mechanisms. J. Fluid Mech. 221, 553-576.

Cabeza, C., Sarasua, G., Marti, A. C., Bove, I., Varela, S., Usera, G. \& Vernet, A. 2010 Influence of coaxial cylinders on the vortex breakdown in a closed flow. Eur. J. Mech. (B/Fluids) 29 (3), 201-207.

Dusting, J., Sheridan, J. \& Hourigan, K. 2006 A fluid dynamics approach to bioreactor design for cell and tissue culture. Biotechnol. Bioengng 94 (6), 1196-1208.

ESCUDIER, M. P. 1984 Observations of the flow produced in a cylindrical container by a rotating endwall. Exp. Fluids 2, 189-196.

Fujimura, K., Koyama, H. S. \& Hyun, J. M. 1997 Time-dependent vortex breakdown in a cylinder with a rotating lid. Trans. ASME: J. Fluids Engng 119 (2), 450-453.

Fujimura, K., Koyama, H. S. \& HyUn, J. M. 2004 An experimental study on vortex breakdown in a differentially-rotating cylindrical container. Exp. Fluids 36, 399-407.

Griffith, M. D., Leweke, T., Thompson, M. C. \& Hourigan, K. 2009 Pulsatile flow in stenotic geometries: flow behaviour and stability. J. Fluid Mech. 622, 291-320. 
Herrada, M. A. \& Shtern, V. $2003 a$ Control of vortex breakdown by temperature gradients. Phys. Fluids 15, 3468-3477.

Herrada, M. A. \& Shtern, V. 2003b Vortex breakdown control by adding near-axis swirl and temperature gradients. Phys. Rev. E 68 (41), 2021-2028.

Hourigan, K., Graham, L. W. \& Thompson, M. C. 1995 Spiral streaklines in pre-vortex breakdown regions of axisymmetric swirling flows. Phys. Fluids 7 (12), 3126-3128.

Husain, H. S., Shtern, V. \& Hussain, F. 2003 Control of vortex breakdown by addition of near-axis swirl. Phys. Fluids 15, 271-279.

Ismadi, M.-Z. P., Meunier, P., Fouras, A. \& Hourigan, K. 2011 Experimental control of vortex breakdown by density effects. Phys. Fluids 23 (3), 034104.

KARniadakis, G. E. \& Sherwin, S. J. 1999 Spectral/hp Element Methods for CFD, 1st edn. Oxford University Press.

Keller, J. J., Egli, W. \& EXLEy, J. 1985 Force- and loss-free transitions between flow states. Z. Angew. Math. Phys. 36, 854-889.

LeWeKe, T., Thompson, M. C. \& Hourigan, K. 2004 Vortex dynamics associated with the impact of a sphere with a wall. Phys. Fluids 16 (9), L74-L77.

Liow, K. Y. S., TAN, B. T., Thouas, G. \& Thompson, M. C. 2009 CFD modelling of the steady-state momentum and oxygen transport in a bioreactor that is driven by a rotating disk. Mod. Phys. Lett. B 23 (2), 121-127.

Lo Jacono, D., Sørensen, J. N., Thompson, M. C. \& Hourigan, K. 2008 Control of vortex breakdown in a closed cylinder with a small rotating rod. J. Fluids Struct. 24 (8), $1278-1283$.

Lopez, J. M., Cui, Y. D., Marques, F. \& LiM, T. T. 2008 Quenching of vortex breakdown oscillations via harmonic modulation. J. Fluid Mech. 599, 441-464.

Meunier, P. \& Hourigan, K. 2013 Mixing in a vortex breakdown flow. J. Fluid Mech. 731, 195-222.

MunungA, L. 2005 Confined flow vortex breakdown study and modelling of mixing in a stirred vessel. PhD thesis, Department of Mechanical Engineering, Monash University, Melbourne, Australia.

Mununga, L., Hourigan, K., Thompson, M. C. \& Leweke, T. 2004 Confined flow vortex breakdown control using a small disk. Phys. Fluids 16 (12), 4750-4753.

OKulov, V. L., Sørensen, J. N. \& Voigt, L. K. 2004 Vortex scenario and bubble generation in a cylinder cavity with rotating top and bottom. Eur. J. Mech. (B/Fluids) 24, 137-148.

Piva, M. \& Meiburg, E. 2005 Steady axisymmetric flow in an open cylindrical container with a partially rotating bottom wall. Phys. Fluids 17 (6), 063603.

Roesner, K. G. 1990 Recirculating zones in a cylinder with rotating lid. Eur. J. Mech. (B/Fluids) 24, 137-148.

RONNENBERG, B. 1977 Ein selbstjustierendes 3-Komponenten-Laserdoppleranemometer nach dem Vergleichsstrahlverfahren, angewandt für Untersuchungen in einer stationären zylindersymmetrischen Drehströmung mit einem Rückströmgebiet. Tech. Rep., MaxPlanck-Institut für Strömungsforschung.

Shtern, V. N., Del Mar Torregrosa, M. \& Herrada, M. A. 2012 Effect of swirl decay on vortex breakdown in a confined steady axisymmetric flow. Phys. Fluids 24, 043601.

Spall, R. E., GAtski, T. B. \& GResch, C. E. 1987 A criterion for vortex breakdown. Phys. Fluids 30, 3434-3440.

SQuire, H. B. 1960 Analysis of the 'vortex-breakdown' phenomenon. Part 1. Imperial College, Aero Department Report 102.

Tan, B. T., Liow, K. Y. S., Mununga, L., Thompson, M. C. \& Hourigan, K. 2009 Simulation of the control of vortex breakdown in a closed cylinder using a small rotating disk. Phys. Fluids 21 (2), 024104.

Thompson, M. C., Leweke, T. \& Hourigan, K. 2007 Sphere-wall collision: vortex dynamics and stability. J. Fluid Mech. 575, 121-148.

Thompson, M. C. \& Hourigan, K. 2003 The sensitivity of steady vortex breakdown bubbles in confined cylinder flows to rotating lid misalignment. J. Fluid Mech. 496, 129-138. 
Thompson, M. C., Hourigan, K., Cheung, A. \& Leweke, T. 2006 Hydrodynamics of a particle impact on a wall. Appl. Math. Model. 30 (11), 1356-1369.

Thompson, M. C., Hourigan, K. \& Sheridan, J. 1996 Three-dimensional instabilities in the wake of a circular cylinder. Exp. Therm. Fluid Sci. 12, 190-196.

Thouas, G. A., Sheridan, J. \& Hourigan, K. 2007 A bioreactor model of mouse tumor progression. J. Biomed. Biotechnol. 9, 32754.

VAlentine, D. T. \& JAhnKe, C. C. 1994 Flows induced in a cylinder with both end walls rotating. Phys. Fluids 16, 2702-2710.

Ventikos, Y. 2002 The effect of imperfections on the emergence of three-dimensionality in stationary vortex breakdown bubbles. Phys. Fluids 14 (3), L13-L16.

Vogel, H. U. 1968 Experimentelle Ergebnisse über die laminare Strömung in einem zylindrischen Gehäuse mit darin rotierender Scheibe. Tech. Rep., Max-Planck Institute.

YU, P., LEE, T. S., ZENG, Y. \& LOW, H. T. $2005 a$ Effect of vortex breakdown on mass transfer in a cell culture bioreactor. Mod. Phys. Lett. B 19, 1543.

YU, P., LEE, T. S., ZENG, Y. \& Low, H. T. $2005 b$ Fluid dynamics of a micro-bioreactor for tissue engineering. Fluid Dyn. Mater. Proc. 1, 235.

YU, P., LEE, T. S., ZENG, Y. \& LOW, H. T. 2006 Effects of conical lids on vortex breakdown in an enclosed cylindrical chamber. Phys. Fluids 18, 117101.

Yu, P., LEE, T. S., ZENG, Y. \& LOW, H. T. 2007 Characterization of flow behaviour in an enclosed cylinder with a partially rotating end wall. Phys. Fluids 19 (5), 057104.

YU, P., LEE, T. S., ZENG, Y. \& LOW, H. T. 2009 Fluid dynamics and oxygen transport in a micro-bioreactor with a tissue engineering scaffold. Intl J. Heat Mass Transfer 52, 316-327. 